\title{
RAPID ESTROGEN AND PROGESTERONE SIGNALING TO DENDRITIC SPINE FORMATION VIA CORTACTIN/WAVE1-ARP2/3 COMPLEX
}

Ivonne Denise Uzair, Marina Ines Flamini, Angel Matias Sanchez*

Laboratory of Signal Transduction and Cell Movement. Institute of Medicine and Experimental Biology of Cuyo (IMBECU). National Scientific and Technical Research Council (CONICET), Mendoza, Argentina.

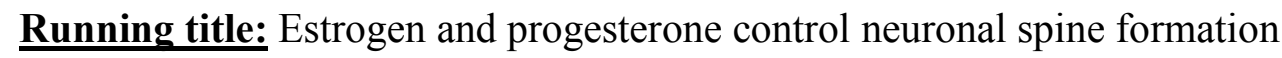

*Address correspondence and reprint requests to:

Angel Matias Sanchez, Ph.D.

Laboratory of Signal Transduction and Cell Movement. Institute of Medicine and Experimental Biology of Cuyo (IMBECU). National Scientific and Technical Research Council (CONICET).

Av. Ruiz Leal s/n. Parque Gral. San Martin CC855, 5500 Mendoza, Argentina.

Phone: 54-261-5244192/4173. Fax: +54-261-5244001

E-mail: amsanchez@mendoza-conicet.gov.ar

Keywords: Estradiol, Progesterone, cortactin, WAVE1, dendritic spines, synaptic plasticity

This work has been supported by National Scientific and Technical Research Council (CONICET) grant PIP 2013-2015 and by the National Agency for Scientific and Technological Promotion (The Ministry of Science, Technology and Productive Innovation) PICT-2013-3230 to A.M.S.

DISCLOSURE STATEMENT: The authors have nothing to disclose. 


\section{ABSTRACT}

Background: Synaptic plasticity is the neuronal capacity to modify the function and structure of dendritic spines (DS) in response to neuromodulators. Sex steroids, particularly $17 \beta$-estradiol (E2) and progesterone (P4), are key regulators in the control of DS formation through multiprotein complexes including WAVE1 protein, and are thus fundamental for the development of learning and memory. Objectives: The aim of this work was to evaluate the molecular switch Cdk5 kinase/PP2A phosphatase in the control of WAVE1 protein (phosphorylation/dephosphorylation) and the regulation of WAVE1 and cortactin to the Arp2/3 complex, in response to rapid treatments with E2 and P4 in cortical neuronal cells. Results: Rapid treatment with E2 and P4 modified neuronal morphology and significantly increased the number of DS. This effect was reduced by the use of a Cdk5 inhibitor (Roscovitine). In contrast, inhibition of PP2A with PP2A DN construct significantly increased DS formation, evidencing the participation of kinase/phosphatase in the regulation of WAVE1 in DS formation induced by E2 and P4. Cortactin regulates DS formation via Src and PAK1 kinase induced by E2 and P4. Both cortactin and WAVE1 signal to Arp2/3 complex to synergistically promote actin nucleation. Conclusion: These results suggest that E2 and P4 dynamically regulate neuron morphology through non-genomic signaling via cortactin/WAVE1-Arp2/3 complex. The control of these proteins is tightly orchestrated by phosphorylation, where kinases and phosphatases are essential for actin nucleation and, finally, DS formation. This work provides a deeper understanding of the biological actions of sex steroids in the regulation of DS turnover and neuronal plasticity processes. 


\section{INTRODUCTION}

Pyramidal neurons from cortex and hippocampus, the main brain areas of language and memory development, bear thousands of dendritic spines (DS) [1]. DS are microscopic protrusions that constitute the main reception point for excitatory synapses in these neurons. As a consequence, failures in DS formation lead to improper cognition and abnormal brain function [2]. In the last few years, it has been demonstrated that the sex steroid hormones $17-\beta$-estradiol (E2) and progesterone (P4) act as neuromodulators and control multiple vital functions, among them learning and memory [3]. Both hormones can activate signaling pathways that ultimately regulate DS formation, contributing to synaptic plasticity [4-7]. The lack of E2 and P4 could therefore be harmful for brain functions. Among others, they could specifically affect learning, cognition, memory and lead to the progression of degenerative diseases, such as Alzheimer's disease [8-10].

Actin filaments are the main cytoskeleton component in DS formation that ultimately will allow synapse formation [11]. Consequently, DS formation depends on the actin cytoskeleton and its regulatory proteins. The Arp $2 / 3$ complex plays a main role in the formation of branched actin filaments, leading to membrane protrusion and new DS [11]. The Arp2/3 complex is controlled by the Wiskott-Aldrich Syndrome Protein family member, verprolin-homologous protein 1 (WAVE1), and cortactin proteins, both known as nucleation promoter factors (NPFs) [12]. In this scenario, reversible protein phosphorylation plays a key role in rapid cellular responses, such as the activation of these actin regulator proteins that enhance new DS formation $[13,14]$. Recent studies have demonstrated that protein phosphatase 2A (PP2A) could dephosphorylate WAVE1, affecting its function and consequently impairing the formation of DS [15]. PP2A is one of the major protein phosphatases in the brain that regulates signaling pathways directly related with actin-based 
structures [16]. Its deregulation has been implicated in many degenerative diseases [17], making its study of great interest.

Cortactin has been proposed to be fundamental for dendritic spine morphogenesis [18]. This scaffolding protein is a controller of the actin cytoskeleton. It is able to regulate actin nucleation by activating the Arp2/3 complex synergistically with WASP proteins, including WAVE1 $[19,20]$.

During our previous work, we have established that E2 and P4 are important cognitive neuromodulators that control rapid signaling pathways leading to the phosphorylation of WAVE1 by ER-PR/Gai-G $\beta / \mathrm{Src} / \mathrm{Rac} 1 / \mathrm{Cdk} 5$ signaling cascades enhancing neuronal spine formation $[21,22]$. In this study we aimed to evaluate if rapid treatments with E2 and P4 control the molecular switch Cdk5 kinase/PP2A phosphatase in the regulation of WAVE1 via phosphorylation/dephosphorylation, key in DS formation. Furthermore, we wished to identify the signaling pathway triggered by cortactin that reinforces the signal of WAVE1 required for Arp2/3 complex activation and strengthen DS formation in cortical neuronal cells. Our results increase our understanding of the complex actions of sex steroids on the control of brain physiology. They may provide new tools for endocrine therapies of key neurological diseases related to age. 


\section{MATERIALS AND METHODS}

\section{Primary Culture of Rat Embryonic Cortical Neurons}

Primary cultures of cortical neurons were obtained from embryonic day 18 rat fetuses as described [23]. This methodology provides a valuable model for investigating synaptic plasticity. Cortical cells were dissected with $0.025 \%$ Trypsin-EDTA (Gibco, Thermo Fisher Scientific, Inc.) for $10 \mathrm{~min}$ at $37^{\circ} \mathrm{C}$ and then dissociated. Cells were seeded on six well culture dishes pre-coated with PEI (polyethyleneimine, $2 \%$ in Borate Buffer $\mathrm{pH} 8.3$ ) at a density of $0.5-1 \times 10^{5}$ cells $/ \mathrm{cm}^{2}$. Neurons were grown in Neurobasal medium (Invitrogen) supplemented with $10 \mathrm{U} / \mathrm{ml}$ penicillin, $10 \mu \mathrm{g} / \mathrm{ml}$ streptomycin, $0.5 \mathrm{mM}$ glutamine, $25 \mu \mathrm{M}$ glutamate, and 2\% B27 (Invitrogen) and kept in vitro for 10-12 days. These culture conditions generate cultures that are $>95 \%$ neuronal and $\leq 5 \%$ glial [24]. Animal maintenance and handling were conducted according to the NIH guide for the Care and Use of Laboratory Animals (NIH publication No 86-23, revised 1985 and 1991) and the UK requirements for ethics of animal experimentation (Animals Scientific Procedures, Act 1986). All experimental procedures were approved by the Care and Use of Laboratory Animals Committee (CICUAL) of the Faculty of Medical Sciences, National University of Cuyo (Protocol approval Nº 33/2014), Mendoza, Argentina.

Progesterone, 17ß-estradiol and Forskolin $(1 \mu \mathrm{M})$ were from Sigma-Aldrich; PP2 (Src inhibitor, $10 \mu \mathrm{M})$ and Roscovitine3 (Cdk5 inhibitor, $50 \mu \mathrm{M})$ were from Calbiochem; FAK inhibitor (FAKi, $(1 \mu \mathrm{M})$, IPA-3 (PAK1 inhibitor, 10 $\mu \mathrm{M})$ and CK-666 (Arp2/3 complex inhibitor, $4 \mu \mathrm{M})$ were from Santa Cruz Biotechnology. ICI 182,780 (100 nM) was obtained from Tocris Cookson (Avonmouth, UK). ORG31710 $(1 \mu \mathrm{M})$ was obtained from Organon Akzo Nobel (Oss, The Netherlands). Whenever an inhibitor was used, the compound was added 30-45 minutes before starting the treatments. 


\section{Immunoblottings}

Cell lysates were separated by SDS-PAGE in $8-10 \%$ gels and transferred into PVDF membranes. Antibodies used were: p-FAK (Y397), Arp3 (612135) (BD Transduction Laboratories); Actin (C-11), Cdk5 (sc-56278), p-Cdk5 (Tyr $\left.{ }^{15}\right)$ (sc-12918), PP2A (sc-13601), p-PP2A (Tyr $\left.{ }^{307}\right)$ (sc-112615), p-FAK (Tyr ${ }^{397}$ ) (sc-11765-R), paxillin (sc-31010), p-paxillin $\left(\mathrm{Tyr}^{118}\right)$ (sc-365020), p-Rac1 (sc-135641), p-PAK1 (Thr $\left.{ }^{423}\right)$ (sc-135755), cortactin (sc11408), p-cortactin (Tyr ${ }^{466}$ ) (sc-101661) (Santa Cruz Biotechnology); p-Src (Tyr ${ }^{418}$ ) (ab4816) (Abcam); p-Arp2 (Thr ${ }^{237}$ ) (orb-155730) (Biorbyt); WAVE1 (SAB4503508), pWAVE1 (S397) (W2768) (Sigma-Aldrich, Saint-Louis, MO); Cdk5 (268-283) (Calbiochem, La Jolla, CA). Primary and secondary antibodies were incubated with the membranes using standard techniques. Immunodetection was accomplished using enhanced chemiluminescence and recorded with a quantitative digital imaging system (Chemidoc XRS with Image Lab, Bio-Rad, USA).

\section{Cell immunofluorescence}

Neuronal cells were grown on coverslips, pre-treated with PEI or Poly-D-Lysine as described above and maintained in Neurobasal medium. Cells were fixed with 4\% paraformaldehyde for 20 minutes and permeabilized with $0.1 \%$ Triton for 5 minutes. Blocking was performed with PBS containing 1\% bovine serum albumin for $30 \mathrm{~min}$ at room temperature (RT). Between each step, cells were carefully washed with PBS two times.

Cells were incubated with antibodies against synaptophysin (1:100, Santa Cruz Biotechnology), PSD-95 (sc-32290) (1:100, Santa Cruz Biotechnology) or p-cortactin ${ }^{\text {Y466 }}$ (1:50) overnight in a humidified chamber at $4^{\circ} \mathrm{C}$, followed by incubation with Alexa Fluor ${ }^{\mathrm{TM}} 350$ (1:250, Invitrogen) or Alexa Fluor ${ }^{\mathrm{TM}} 488$ (1:300, Invitrogen) secondary 
antibody at RT for $2 \mathrm{~h}$. Cells were then incubated with Texas Red-phalloidin (SigmaAldrich) for 30 minutes at RT to visualize actin filaments. After washing, the nuclei were counterstained with 4'-6-diamidino-2-phenylindole (DAPI) (Sigma-Aldrich, Saint-Louis, MO) and mounted with Vectashield mounting medium (Vector Laboratories, Burlingame, CA). Immunofluorescence was visualized using a Nikon Eclipse E200 microscope and recorded with a high-resolution DP70 Olympus digital camera.

\section{Dendritic spines quantification}

In order to determine the dendritic spine classification after $(10 \mathrm{nM}, 20 \mathrm{~min}) \mathrm{E} 2$ and P4 treatments, images of immunostained neurons were visualized with a Nikon Eclipse E200 microscope. Two dendrites of each neuron were chosen at random, and within each dendrite a $10 \mu \mathrm{m}$ extension region was sampled and dendritic spines were counted. For morphological classification of spines, three subtypes were considered: 1) mushroom spines were those with a wide head and defined neck, 2) thin spines were those with a long and thin defined neck but without a head, and 3) stubby spines were small and wider protrusions without a defined neck, according to the guidelines of [25].

\section{Co-immunoprecipitation assay}

Cortical neuronal cells were washed with ice-cold PBS and lysed with $20 \mathrm{mM}$ Tris- $\mathrm{HCl}(\mathrm{pH}$ 7.4), $10 \mathrm{mM}$ EDTA, $100 \mathrm{mM} \mathrm{NaCl}, 1 \%$ Igepal, $1 \mathrm{mM} \mathrm{Na} \mathrm{VO}_{4}, 50 \mathrm{mM} \mathrm{NaF}, 0.1 \mathrm{mg} / \mathrm{L}$ PMSF, $0.3 \mathrm{mg} / \mathrm{L}$ aprotinin and $0.01 \%$ protease inhibitor mixture (Sigma-Aldrich, SaintLouis, MO). Immunoprecipitating antibody against Arp3 (BD Transduction Laboratories) in $500 \mu \mathrm{l}$ of lysis buffer was then added and incubated for $1 \mathrm{~h}$ at $4^{\circ} \mathrm{C}$ with gentle rocking. $40 \mu \mathrm{l}$ of 1:1 Protein-A-agarose (Santa Cruz Biotechnology) was then added and gently rocked for 
$2 \mathrm{~h}$ at $4^{\circ} \mathrm{C}$. The mixture was centrifuged at $12,000 \times \mathrm{g}$ for $5 \mathrm{~min}$ at $4^{\circ} \mathrm{C}$. The supernatant was removed and the immunoprecipitates washed with $500 \mathrm{ml}$ of $20 \mathrm{mM}$ Tris- $\mathrm{HCl}(\mathrm{pH} 7.4), 10$ mM EDTA, $150 \mathrm{mM} \mathrm{NaCl}$, 1\% IGEPAL, $1 \mathrm{mM} \mathrm{Na}_{3} \mathrm{VO}_{4}, 50 \mathrm{mM} \mathrm{NaF}, 0.1 \mathrm{mg} / \mathrm{L}$ PMSF, 0.3 $\mathrm{mg} / \mathrm{L}$ aprotinin and $0.01 \%$ protease inhibitor mixture (Sigma-Aldrich, Saint-Louis, MO). Immunoprecipitated proteins were separated under reducing and denaturing conditions by $10 \%$ SDS-PAGE and transferred to a polyvinylidene difluoride membrane. Non-specific binding was blocked with 3\% BSA (Bovine serum albumin) in PBS-Tween 20. Membranes were incubated with anti-cortactin and Arp3 antibodies.

\section{Gene silencing with RNA interference}

Synthetic small interfering RNAs targeting WAVE-1 (siRNA SMARTpool WAVE-1) and control siRNAs (D-001810-01-05) were purchased from Dharmacon (Thermo Fisher Scientific Inc). Paxillin siRNAs and control siRNAs were purchased from Santa Cruz Biotechnology (Santa Cruz Biotechnology). The siRNAs were used at a final concentration of 50-75 nM. Cortical neurons were treated $48 \mathrm{~h}$ after siRNAs transfection. Efficacy of gene silencing was checked with Western analysis and found to be optimal at $48 \mathrm{~h}$.

\section{Transfection experiments}

A mutant construct for cortactin (cortactin ${ }^{3 Y F}$, a mutant form of cortactin with Tyr changed to Phe, which prevents cortactin phosphorylated in $\mathrm{Y}^{421 / 466 / 482}$ by Src) was generously provided by Dr. John Cooper (Washington University School of Medicine, USA). The insert was cloned in a pcDNA 2AB Flag-cortactin 3YF [26]. A phosphorylation mimic mutant of PP2A $\left(P P 2 A^{Y 307 E}\right)$ and a non-phosphorylatable mutant of PP2A $\left(P P 2 A^{Y 307 F}\right)$ were a kind gift from Dr. David C. Pallas (Winship Cancer Institute, Emory University School of Medicine, Atlanta, USA). The dominant-negative construct for Rac1 ( $\left.R a c 1^{T 17 N}\right)$ was from the Guthrie 
cDNA Resource Center (www.cdna.org). The inserts were cloned in pcDNA3.1+. The plasmids $(10 \mu \mathrm{g})$ were transfected into cortical neurons using Lipofectamine (Invitrogen, Carlsbad, CA). Parallel cells were transfected with empty pcDNA3.1+ plasmid. Cells $(60-$ $70 \%$ confluent) were treated $24-48 \mathrm{~h}$ after transfection.

\section{Statistical analysis}

All values are expressed as (mean \pm SD) of three independent experiments. Statistical analysis of the data was performed using one-way analysis of variance (ANOVA) followed by Dunnett's multiple comparisons test with GraphPad Prism 5 software. $\mathrm{P}<0.05$ was considered as statistically significant. 


\section{$\underline{\text { RESULTS }}$}

\section{$\underline{\text { Rapid treatments with } \mathrm{E} 2 \text { and P4 increase thin dendritic spine formation }}$}

We have previously reported that treatments with $17 \beta$-estradiol (E2) and progesterone (P4) act as potent neuromodulators of cortical neuronal dendritic architecture [21, 22]. To continue investigating the molecular mechanisms that regulate the control of DS formation, we evaluated the rapid action of E2 $(10 \mathrm{nM})$ and P4 $(10 \mathrm{nM})$ on cortical neuronal cells (Fig. 1A-C). By immunofluorescence, we observed that E2 and P4 exposure for 20 min rapidly modifies neuron morphology, significantly increasing DS density compared to control cells (Fig. 1A-B). To further investigate the morphological classification of spines induced by E2 and P4 in cortical neuronal cells, we quantified three typical DS morphologies, mushroom, stubby and thin, in cells treated with $\mathrm{E} 2(10 \mathrm{nM}, 20 \mathrm{~min})$ and P4 (10 nM, $20 \mathrm{~min})$. This treatment significantly increased the number of thin spines compared to control conditions (Fig. 1C). Thin spines are first formed during spinogenesis. They are able to become into mature spines by acquiring a mushroom morphology during synaptic plasticity processes [27]. These results suggest that E2 and P4 promote the rapid development of thin spines, modulating the early rearrangement of DS structure and contributing to synaptic plasticity processes.

To evaluate whether E2 and P4 increase synapse formation and the implications of rapid and transient nature of the structural changes induced by E2 and P4, we used the pre- and postsynaptic markers synaptophysin and PSD-95, respectively (Figure 1D-I). We observed that exposure to E2 and P4 during 20 min significantly increased the density of synaptophysin (Figure 1D-F) and PSD-95 (Figure 1E-G), as well as the number of dendritic spines containing synaptophysin and PSD-95 (Figure 1H-I). 


\section{Estrogen and progesterone modulate DS formation through Src/FAK/paxillin/Rac1 in}

\section{$\underline{\text { neuronal cells }}$}

We have previously demonstrated that Src and focal adhesion kinases (FAK) are fundamental in the signaling cascade and responsible for enhancing neuronal dendritic spine formation via WAVE1 [21, 22]. Other authors have described that both proteins recruit paxillin, a structural cytoskeleton component necessary for dendritic spine morphogenesis [28]. For this reason, we wanted to evaluate if these proteins are involved in the previously described signaling pathway. The Src kinase inhibitor (PP2, $10 \mu \mathrm{M})$ and the specific FAK inhibitor $(\mathrm{FAKi}, 1 \mu \mathrm{M})$ blocked $\mathrm{FAK}^{\mathrm{Y} 397}$ and paxillin ${ }^{\mathrm{Y} 118}$ phosphorylation induced by E2 and P4 (Fig. 2A-C).

In order to clarify whether Rac1 GTPase could mediate the signaling of E2 and P4 to WAVE1 and cortactin, we blocked paxillin with small interfering RNAs and Rac1 with dominant negative constructs. Paxillin silencing impaired both paxillin ${ }^{\mathrm{Y} 118}$ and $\mathrm{Rac1}^{\mathrm{S} 71}$ phosphorylation in the presence of E2 and P4 (Fig. 2D-F). Blockade of Rac1, however, impaired Rac1 phosphorylation by E2 or P4, but not of paxillin (Fig. 2D-F), supporting the concept that E2 and P4 signal to Src/FAK/paxillin/Rac1 cascade leading to DS formation. As control, we observed that paxillin expression was significantly reduced when cortical neuronal cells were transfected for $24 \mathrm{~h}$ with paxillin siRNA (Fig. 2G-H), whereas cells transfected with the dominant negative mutant of Rac1 showed increased Rac1 expression (Fig. 2I-J).

By immunofluorescence we visualized that the inhibition of Src (PP2), FAK (FAKi) and paxillin (siRNAs) abolished the increase in spine density triggered by the treatment with E2 (20 min) and P4 (20 min) (Fig. 2K-L). 


\section{E2 and P4 induce DS formation via WAVE1 through the molecular switch Cdk5}

\section{$\underline{\text { kinase/PP2A phosphatase }}$}

We have previously shown that estrogen and progesterone trigger a rapid phosphorylation of Cdk5 through Rac1 GTPases and promote the formation of DS in rat cortical neurons [21, 22]. Ceglia et al. have demonstrated that the molecular switch Cdk5 kinase/PP2A phosphatase controls WAVE1 phosphorylation and, consequently, DS formation [15], but the action of E2 and P4 on this switch remains to be elucidated. Hence, our next step was to evaluate this molecular control. We observed a significant increase in $\mathrm{Cdk} 5^{\mathrm{Y} 15}$ and WAVE1 ${ }^{\mathrm{S} 397}$ phosphorylation during $20 \mathrm{~min}$ of E2 and P4 exposure (Fig. 3A-C). Treatment of neuronal cells with the specific Cdk5 inhibitor Roscovitine resulted in a clear inhibition of $\mathrm{Cdk} 5^{\mathrm{Y} 15}$ and WAVE1 ${ }^{\mathrm{S} 397}$ phosphorylation during exposure to E2 and P4 (Figure 3A-C).

The increase in DS density after treatment with E2 and P4 (Fig. 3D-E) was completely prevented with Roscovitine (Fig. 3D-E). This result suggests that E2 and P4 induce WAVE1 phosphorylation and lead to DS formation through Cdk5 kinase.

According to studies performed by Chen Jian and collaborators, phosphatase PP2A activity is regulated by tyrosine phosphorylation on residue 307 [29]. We therefore analyzed the effect of E2 and P4 on phosphorylation levels of PP2A and WAVE1 and, consequently, DS formation. We observed that both hormones significantly increase the inhibitory phosphorylation levels of $\mathrm{PP} 2 \mathrm{~A}^{\mathrm{Y} 307}$ (Figure $3 \mathrm{~F}-\mathrm{G}$ ), in parallel with WAVE1 $1^{\mathrm{S} 397}$ phosphorylation (Figure $3 \mathrm{~F}$ and $\mathrm{H}$ ). Treatments with the PP2A activator Forskolin, significantly reduce the inhibitory phosphorylation levels of $\mathrm{PP} 2 \mathrm{~A}^{\mathrm{Y} 307}$ and WAVE1 ${ }^{\mathrm{S} 397}$ (Figure 3F-H) and significantly abolish the increase of DS induced by E2 and P4 (Figure 3IJ). These results suggest that E2 and $\mathrm{P} 4$ regulate $\mathrm{PP} 2 \mathrm{~A}$ activity by increasing $\mathrm{PP} 2 \mathrm{~A}^{\mathrm{Y} 307}$ phosphorylation and thus inactivating it, which leads to an increased WAVE1 
phosphorylation and promotes DS formation. Reducing $\mathrm{PP} 2 \mathrm{~A}^{\mathrm{Y} 307}$ phosphorylation with Forskolin results in WAVE1 dephosphorylation and a reduction in DS formation triggered by E2 and P4.

\section{PP2A phosphatase regulates WAVE1 and DS formation induced by estrogen and}

\section{progesterone}

In the figure 3, we have shown that PP2A is an important component of the regulation mechanism of WAVE1 and, consequently, of the formation of DS induced by E2 and P4. As a next step, we aimed to investigate whether the inhibitory phosphorylation of PP2A ( $\left.\mathrm{Tyr}^{307}\right)$ could be responsible for the dephosphorylation of WAVE1 and DS formation. We transfected cortical neurons with two mutant constructs of PP2A, $P P 2 A^{Y 307 E}$ that mimics a constitutive phosphorylation in the $\mathrm{C}$ subunit of PP2A and therefore acts as dominant negative $(\mathrm{DN})$ construct, and $P P 2 A^{Y 307 F}$ that cannot become phosphorylated and thus acts as a constitutively active (CA) form of the protein. The transfections with both plasmids increased the expression of the catalytic subunit of PP2A that contains the specific residue $\left(\mathrm{Tyr}^{307}\right.$ ) (Figure 4A-B). In addition, the transfections with $P P 2 A^{Y 307 E}$ resulted in an increase in WAVE1 ${ }^{\mathrm{S} 397}$ phosphorylation (Figure 4A-C) and DS density (Figure 4D-E), while the use of the CA construct impaired these effects (Figure 4A,C and D-E), suggesting a specific role of PP2 $\mathrm{A}^{\mathrm{Y} 307}$ in the control of WAVE1 and DS formation induced by E2 and P4.

Phosphatase PP2A interacts with Src kinase, leading to its inactivation $[30,31]$. We therefore evaluated whether Src is involved in the control of PP2A phosphorylation by using the specific Src inhibitor (PP2). We demonstrated that E2 and P4 induced $\mathrm{Src}^{\mathrm{Y} 418}$ and PP2 $\mathrm{A}^{\mathrm{Y} 307}$ phosphorylation. This effect was prevented by PP2, suggesting a crucial role of Src in PP2A phosphorylation (Figure 4F-H). In parallel, blockade of Src with PP2 
completely abolished E2 and P4-dependent $\operatorname{cortactin}^{\mathrm{Y} 466}$ phosphorylation (Fig. 4F and I), suggesting that Src acts as a central protein between PP2A and cortactin, modulating the signal that promote the DS formation in cortical neurons.

\section{Cortactin participates in the E2 and P4 signaling to the formation of dendritic spines}

\section{via Src and Rac1/PAK1 in cortical neurons}

Previous studies have provided evidence that paxillin signals to PAK1 serine/threonine kinase, an effector of Rac1 GTPases. When PAK1 is phosphorylated, it may allow the recruitment of cortactin to promote the development of protrusive membrane structures [32, 33], a mechanistic process that shares similarities with DS formation [34]. Cortactin is regulated by several proteins like Src and PAK1 that modulate its function via specific phosphorylations [35]. Src tyrosine kinase targets tyrosine residues $\mathrm{Y}^{421 / 466 / 482}$, while PAK1 phosphorylates serine $\mathrm{S}^{113}[36,37]$. We demonstrated that in Rac1-transfected cells, phosphorylation of $\mathrm{Rac}^{\mathrm{S} 71}$ and $\mathrm{PAK} 1^{\mathrm{T} 423}$ was significantly lower after E2 and P4 treatment, whereas the inhibition of PAK1 with the specific inhibitor IPA-3 lead to a decrease in PAK1 ${ }^{\mathrm{T} 423}$ phosphorylation but did not affect the Rac1 ${ }^{\mathrm{S} 71}$ phosphorylation in cortical neuronal cells (Fig. 5A and C), suggesting that E2 and P4 signal to Rac1 and PAK1 to finally promote cortactin phosphorylation.

Cortactin has been previously described to be concentrated in dendritic spine $[18,38]$. We therefore evaluated the distribution of phosphorylated cortactin $^{\mathrm{Y} 466}$ in dendritic protrusions of cortical neurons after treatment with E2 and P4. In control cells, phospho-cortactin ${ }^{\mathrm{Y} 466}$ was diffusely distributed throughout the dendrites (Fig. 5D). Treatment for 20 min with E2 and P4 induced an increase in phospho-cortactin ${ }^{\mathrm{Y} 466}$ levels and its translocation to the edge of the dendrites, mainly where spines were formed (Fig. 5D-E). The redistribution of 
phospho-cortactin ${ }^{\mathrm{Y} 466}$ was impaired by the blockade of cells expressing a cortactin mutant that prevents cortactin phosphorylation in the residue Y466 (cortactin ${ }^{3 Y F}$ ) (Figure 5D-E), induced by Src (Fig. 4F and I).

To test the specific role of Rac1, PAK1 and cortactin on the control of DS formation, we performed immunofluorescence assay (Figure 5F-G). The increase in DS formation induced by E2 and P4 was abolished after the blockade of Rac1 (Rac1 $\left.{ }^{\mathrm{T} 17 \mathrm{~N}}\right)$, PAK1 (IPA-3) and the transfection of a non-phosphorylatable construct of cortactin ( $\operatorname{cortactin}^{3 \mathrm{YF}}$ ) in neuron cells (Figure 5F-G), suggesting that $\mathrm{Src}$ and Rac1/PAK1 are fundamental in the cortactin function, via specific phosphorylations, modulating the control of DS formation triggered by E2 and P4 (Fig. 5A-G and Fig. 4F and I). These results highlight the important role of cortactin as a key component in the control of DS formation regulated by E2 and P4 in neuronal cells.

\section{Estrogen and progesterone control Arp2/3 complex through cortactin-dependent}

\section{signaling pathway}

Previous studies have indicated that cortactin interacts with the Arp2/3 complex by binding to Arp3 subunit in vitro [39]. We used immunoprecipitation assays to test the interaction between Arp3 subunit and cortactin protein after treatment with E2 and P4. Both hormones reduced the interaction of cortactin with Arp3 subunit compared to control treatments (Figure 6A-D).

To explain this dissociation we tested whether the pulse with E2 (20 min) and P4 (20 min) could lead to phosphorylation of Arp2 subunit (Arp2/3 complex) and dissociation from cortactin by increasing phosphorylation levels of Arp2, since it has been previously described as a necessary step in Arp2/3 complex activation [40]. This specific 
phosphorylation seems to be fundamental for binding the pointed end of actin filaments and promote the actin nucleation process [41]. We show here that E2 and P4 significantly increase $\operatorname{Arp} 2^{\mathrm{T} 237}$ phosphorylation (Figure $6 \mathrm{E}-\mathrm{H}$ ) and that this effect is prevented by transfection of the cortactin construct (cortactin ${ }^{3 Y F}$ ) (Figure 6E-H), suggesting that E2 and P4 promote Arp $2^{\mathrm{T} 237}$ phosphorylation and the consequent dissociation of cortactin from the Arp2/3 complex.

\section{$\underline{\text { Regulation of Arp2/3 complex by cortactin and WAVE1 in neuron cells }}$}

Actin nucleation by the Arp $2 / 3$ complex is fundamental for the rapid formation of an actin network at the leading edge of the cell [42]. Arp2/3-complex is regulated by upstream NPFs, including cortactin and WAVE1 $[12,43]$. We studied whether the phosphorylation of cortactin and WAVE1 induced by estrogen and progesterone may specifically regulate Arp2/3 complex in neuronal cells. Rapid treatment with E2 (20 min) and P4 (20 min) significantly increased the phosphorylation levels of cortactin $^{\mathrm{Y} 466}$ (Figure 7A and C), WAVE1 $^{\mathrm{S} 397}$ (Figure 7A and D) and $\mathrm{Arp}^{\mathrm{T} 237}$ (Figure 7A and E). The transfection with $\operatorname{cortactin}^{3 Y F}$ (Figure 7A-B) impaired the phosphorylation of $\operatorname{cortactin}^{\mathrm{Y} 466}$ (Figure 7A and C) and $\operatorname{Arp} 2^{\mathrm{T} 237}$ (Figure 7A and E), but did not affect the WAVE1 ${ }^{\mathrm{S} 397}$ phosphorylation induced by E2 and P4 (Figure 7A and D).

In parallel, silencing of WAVE1 with specific siRNAs significantly decreased WAVE1 ${ }^{\text {S397 }}$ (Figure $7 \mathrm{~F}$ and $\mathrm{H}$ ) and $\mathrm{Arp} 2^{\mathrm{T} 237}$ (Figure $7 \mathrm{~F}$ and $\mathrm{J}$ ) phosphorylation induced by both hormones, whereas the cortactin ${ }^{\mathrm{Y} 466}$ phosphorylation was not modified (Figure 7F-I).

Estrogen and progesterone induce the formation of dendritic spines via ER/PR to WAVE1-Arp2/3 complex 
Phosphorylation of Arp2 in Threonine ${ }^{237}$ residue is a key regulatory step for Arp2/3 complex mediated nucleation [41]. We used a specific inhibitor of the Arp2/3 complex (CK-666) to evaluate the specific role of Arp2/3 complex in cortical neurons (Figure 8A-D). Treatment with CK-666 significantly impaired Arp2 $2^{\mathrm{T} 237}$ phosphorylation (Figure 8A-B) and DS formation (Figure 8C-D) during E2 and P4 administration.

Finally, to confirm that the specific phosphorylation of PP2A, WAVE1 and Arp2/3 complex occurs through estrogen (ER) and progesterone receptors (PR), we used the specific ER/PR antagonists ICI 182,780 (100 nM) and ORG $31710(1 \mu \mathrm{M})$, respectively. Blockade of ER and PR completely abolished E2 and P4-dependent PP2A, WAVE1 and Arp2/3 phosphorylation, confirming that E2 and P4 regulate these signals via ER/PR (Figure 8 E-F). 


\section{$\underline{\text { DISCUSSION }}$}

The study of dendritic spine (DS) formation has gained interest in the past few years, especially due to the important role of these structures in establishing excitatory neuronal circuits for memory acquisition and enabling learning and cognition [44]. Neurons from cognitive brain regions like cortex and hippocampus depend on the neuronal plasticity to remodel their connections [45], thorough dynamic actin cytoskeleton polymerization/depolymerization and the development of protrusive membrane structures, such as DS [22].

The sex steroids estrogen and progesterone modify DS density, regulating neuron morphology [4], synaptic plasticity [5] via actin cytoskeleton reorganization, developing DS $[21,22]$. Dendritic spine density in pyramidal neurons of the cortex correlates with circulating estradiol levels [8]. E2 and P4 exert morphological changes in dendrites and synapses, and even modify neurotransmitter levels during periods of marked fluctuations in their levels, such as puberty, reproductive cycles, pregnancy or menopause, [4]. These events depend on the rapid regulation of several proteins that control the actin polymerization/de-polymerization, leading to the formation of new DS.

The major findings of this work is that we demonstrate that E2 and P4 signal to the actin controllers cortactin/WAVE1-Arp2/3 complex and trigger a rapid modification of cell membrane morphology, promoting DS formation and enhancing neuronal plasticity. These events are mediated through two main rapid signaling pathways. One of them is triggered by E2 and P4 to ER-PR/Src/FAK/paxillin. When paxillin is phosphorylated, it recruits Rac1 and Cdk5, leading to WAVE1 ${ }^{\mathrm{S} 397}$ phosphorylation and enhancing DS formation. In the other pathway, E2 and P4 recruit an ER-PR/Src/FAK/paxillin/Rac1/PAK1/cortactin cascade, which leads to cortactin phosphorylation and redistribution to sites where DS are formed. In addition, we provide evidence that Rac1 GTPase is a central protein that controls actin nucleation through both cortactin and WAVE1 via Arp2/3 complex (Figure 9). 
We also observed that E2 and P4 increase the number of thin DS within minutes of treatment, while the quantity of mushroom and stubby shapes remains without changes. Thin DS are highly dynamic and can retract and expand within hours of being stimulated [46]. They are considered a transient phenotype and a good substrate to become a mature spine after increased synaptic inputs $[46,47]$. Here, we also demonstrated that E2 and P4 increase the density and localization of pre- and post-synaptic markers (synaptophysin and PSD-95, respectively) in DS, suggesting that the hormones may stimulate synaptogenesis in cortical neuronal cells. These changes driven by sex hormones involve the formation of DS and their maturation from thin spines to a mushroom phenotype as well as synaptogenesis, which is a crucial aspect of cognitive development [48].

In previous studies we found that E2 and P4, via Estrogen and/or Progesterone Receptors (ER/PR), represent the starting platform to phosphorylate/activate Src and FAK, leading to an increased DS formation in neurons via the small G proteins Gail and $G \beta 1[21,22]$. Here, we continued evaluating the rapid actions of E2 and P4 on diverse kinases and scaffold proteins involved in cell morphology and movement.

Additionally, this study shows that E2 and P4 increase the phosphorylation levels of p$\mathrm{Rac}^{\mathrm{S} 71}$ by regulating the actin cytoskeleton through Src, FAK and paxillin proteins. Rac1 GTPases represent key regulators of actin dynamics. Active Rac1 promotes F-actin polymerization, which leads to axon outgrowth and extension as well as dendritic spine formation through the activation of downstream effectors, such as Cdk5 and PAK1 [2]. We confirm that the phosphorylation of $\mathrm{Rac}^{\mathrm{S} 71}$ is essential for $\mathrm{Cdk} 5$ activation, leading to WAVE1 phosphorylation and increasing DS formation after rapid treatments with E2 and P4. Roscovitine, Cdk5 inhibitor, abrogated this event and this is in agreement with our previous works [21, 22]. 
We describe for the first time that E2 and P4 control WAVE1 phosphorylation by the molecular switch Cdk5 kinase/PP2A phosphatase. PP2A is a major phosphatase with important implications for human health. Deficiencies in its activity have severe health consequences as a result of an unbalanced phosphorylation status [49]. Both, the downregulation and an increased phosphorylation of $\mathrm{PP} 2 \mathrm{~A}^{\mathrm{Y} 307}$ are correlated with hyperphosphorylation of Tau protein in Alzheimer disease [50]. Here we show that E2 and P4 via ER/PR increase $\mathrm{PP} 2 \mathrm{~A}^{\mathrm{Y} 307}$ phosphorylation that leads to an enhanced WAVE1 phosphorylation and DS formation. Reducing $\mathrm{PP}_{2} \mathrm{~A}^{\mathrm{Y} 307}$ phosphorylation with the specific PP2A activator Forskolin causes WAVE1 dephosphorylation and therefore reduces DS formation. The expression of PP2A DN and CA mutants further confirms that the phosphorylation of $\mathrm{PP} 2 \mathrm{~A}^{\mathrm{Y} 307}$ is necessary for DS formation mediated by these hormones. Only a few other research groups have addressed the role of PP2A in DS formation. Li et al. recently indicated that PP2A is necessary for DS formation in striatal neurons [51, 52]. Liu et al. determined that PP2A interacts with the protein PRG-1 at the postsynaptic density not only to modulate synaptic plasticity, but also to produce the recruitment of focal adhesion proteins like Src and paxillin [53]. Here, we determined that E2 and P4 reduce PP2A activity via Src kinase, leading to an increase in WAVE1 phosphorylation and, hence, DS formation (Figure 9). This is supported by several studies that link PP2A with Src kinase family [30, $31]$.

Cortactin is another key NPF enriched in spines and involved in the regulation of DS morphology. A decrease in its expression reduces DS density, whereas its overexpression enhances spine length [18]. Here we propose that E2 and P4 increase cortactin phosphorylation and redistribution near the dendritic protrusions through two pathways, via Src/FAK/paxillin/Rac1/PAK1 and/or directly through Src kinase (Figure 9). PAK1 serine/threonine kinase promotes cortactin phosphorylation on serine residue 113, while Src 
phosphorylates cortactin in Tyr 421, 466 and 482 [36, 37]. Furthermore, we describe that cortactin interacts with Arp3 subunit, and that E2 or P4 disrupts this interaction. We also observed that both hormones increase Arp $2^{\mathrm{T} 237}$ subunit phosphorylation. We confirmed that Arp2 phosphorylation disrupt the interaction between Arp3 and cortactin and thus stabilize the new F-actin branches. Cortactin and WAVE1 interact with the Arp2/3 complex, and both independently regulate actin nucleation [20]. The latter is based on two observations: first, when cortactin phosphorylation is impaired, phosphorylation of Arp2, but not WAVE1, is reduced. Second, the impairment of WAVE1 phosphorylation leads to a reduction in phosphorylation of Arp2, but not of cortactin.

Several works have shown that WAVE1 knockout mice exhibit reduced memory and learning [54], and loss of WAVE1 function in vivo or in cultured neurons results in a reduction in the number of mature dendritic spines [22]. Whereas a reduced cortactin expression has been found in brain tissue from postmortem patients with schizophrenia [55]. On the other hand, loss of Arp2/3 activity in excitatory cortical neurons leads to an asymmetric structural plasticity of DS resulting in a progressive loss of DS spine synapses [56]. Altogether, this evidence suggests that dysfunctions in actin signaling that converge in Arp2/3-mediated nucleation may explain the etiology of neurodegenerative disorders affecting cognition when a loss of estrogens and progestins occurs.

In conclusion, our results highlight that E2 and P4 rapidly exert a tight regulation on Arp2/3 complex activation, orchestrating different branches of signaling pathways that culminate with the phosphorylation of cortactin, WAVE1, and finally Arp2 in order to enhance DS formation in cortical neurons, and thus regulate synaptic plasticity (Figure 9) [21, 22, 41]. Our results are particularly important for women receiving estrogen replacement, as they could potentially prevent or even reverse the age-related decline in learning and memory during menopause. Further investigation in this area will be promising for clinical 
manipulation with a view to future therapy that could help to engineer newer and more selective pharmacologic tools for endocrine therapies against some of the gender-related degenerative changes observed in the brain throughout aging. 


\section{FIGURE LEGENDS}

Figure 1. 17- $\beta$-estradiol (E2) and progesterone (P4) enhance dendritic spine density. A) Cortical neurons were cultured and treated with E2 and P4 (10 nM, $20 \mathrm{~min})$, actin filaments were counterstained with Texas Red-phalloidin and cell nuclei with DAPI. Representative photographs of cortical neuronal cells are shown with immunofluorescence (scale bar $=10$ $\mu \mathrm{m})$. The inserts show dendritic spines at higher magnification from the white boxes (scale bar $=5 \mu \mathrm{m}$ ). The results are derived from the sampling of five areas of the cell membrane of 30 randomly chosen cells. B) Mean $\pm \mathrm{SD}$ of the number of spines in $10 \mu \mathrm{m}$ of dendrite length (20-30 neurons from three independent experiments were analyzed per condition). ${ }^{*} \mathrm{p}<0.05$ vs. control, CON. C) Number of the three typical morphologies of DS, mushroom, stubby and thin, after hormone stimulation (20-30 neurons from three independent experiments). ${ }^{*} p<0.05$ vs. CON. D-E) Representative image of dendritic spines (Texas Redphalloidin) co-stained for synaptophysin (Alexa Fluor ${ }^{\mathrm{TM}} 488$ ) and for PSD-95 (Alexa FluorTM35) markers. F-G) Mean \pm SD of synaptophysin and PSD-95 density vs. CON. HI) Quantitative analysis of the number of overall synaptophysin and PSD-95 puncta (synaptophysin and PSD-95 puncta per $10 \mu \mathrm{m}$ dendrite length) containing spines ( $\mathrm{n}=25$ cells per condition). ${ }^{*} \mathrm{p}<0.05$ vs. CON. The experiments were repeated three times with consistent results. The scale bar corresponds to $10 \mu \mathrm{m}$.

Figure 2. E2 and P4 promote dendritic spine formation via Src/FAK/paxillin/Rac1. Cortical neurons were treated with the specific inhibitor of Src (PP2, $10 \mu \mathrm{M})$ and FAK kinase (FAKi, $1 \mu \mathrm{M}$ ) for 45-60 min before E2 and P4 treatments (10 nM, $20 \mathrm{~min})$. A) Expression of phosphorylated $\mathrm{FAK}^{\mathrm{Y} 397}$ and paxillin ${ }^{\mathrm{Y} 118}$ were analyzed with Western blot assay. B-C) Densitometric analysis of phospho-FAK ${ }^{\mathrm{Y} 397}$ and phospho-paxillin ${ }^{\mathrm{Y} 118}$ bands. 
Intensity values of each phosphorylated protein were adjusted to actin intensity and normalized to the control. ${ }^{*} \mathrm{p}<0.05$ vs. control, CON. D-G-I) Neurons were transfected with specific siRNAs vs. paxillin (siRNA paxillin) and with a Rac1 dominant negative mutant (Rac1DN) for $48 \mathrm{~h}$ before hormone treatments. Paxillin, p-paxillin ${ }^{\mathrm{Y} 118}$, Rac1 and p-Rac1 ${ }^{\mathrm{S} 71}$ were assayed with Western analysis. E-F) Phospho-paxillin ${ }^{\mathrm{Y} 118}$, p-Rac1 ${ }^{\mathrm{S} 71}$ and $\left.\mathbf{H}-\mathbf{J}\right)$ Paxillin, Rac1 densitometry values were adjusted to actin intensity and normalized to the control. ${ }^{*} \mathrm{p}<0.05$ vs. control, CON. K) Dendritic spine density was measured with immunofluorescence after staining actin fibers with Texas Red-phalloidin. The scale bar corresponds to $5 \mu \mathrm{m}$. L) Quantitative analysis of spine density expressed as the number of spines per $10 \mu \mathrm{m}$ dendrite length (20-30 neurons from three independent experiments were analyzed per condition). Results are expressed as the mean \pm S.D. ${ }^{*} p<0.05$ vs. CON. The experiments were performed in triplicates and representative images are shown.

Figure 3. Molecular switch Cdk5/PP2A regulates WAVE1 and DS formation. Neuron cells were exposed for $20 \mathrm{~min}$ to $\mathrm{E} 2(10 \mathrm{nM})$ and $\mathrm{P} 4(10 \mathrm{nM})$ in the presence or absence of the Cdk5 inhibitor (Roscovitine, $50 \mu \mathrm{M}$ ). A) $\mathrm{Cdk} 5$, p-Cdk $5^{\mathrm{Y} 15}, \mathrm{WAVE} 1$ and $\mathrm{p}-\mathrm{WAVE} 1^{\mathrm{S} 397}$ were assayed with Western analysis. Phospho-Cdk5 ${ }^{\mathrm{Y} 15}$ and $\mathrm{p}-\mathrm{WAVE1}{ }^{\mathrm{S} 397}$ densitometry values were adjusted to $\mathrm{Cdk} 5$ and WAVE1 intensity and normalized to the control. CON, Control. B-C) Optical densitometry was performed in triplicate with consistent results; representative images are shown. ${ }^{*} \mathrm{p}<0.05$ vs. CON. D) Dendritic spine formation and number were measured with immunofluorescence after staining actin fibers with Texas Redphalloidin. The scale bar corresponds to $5 \mu \mathrm{m}$. E) Quantitative analysis of spine density expressed as the number of spines per $10 \mu \mathrm{m}$ dendrite length. The results are expressed as the mean \pm S.D. ${ }^{*} \mathrm{p}<0.05$ vs. CON. F) Cortical neurons were treated with PP2A activator Forskolin (1 nM) for $20 \mathrm{~min}$ alone or in combination with E2 and P4. F) Expression of 
phosphorylated $\mathrm{PP} 2 \mathrm{~A}^{\mathrm{Y} 307}$ and $\mathrm{WAVE1}^{\mathrm{S} 397}$ was analyzed with Western blot. G) Densitometric analysis of phospho-PP2 $\mathrm{A}^{\mathrm{Y} 307}$ and phospho-WAVE1 ${ }^{\mathrm{S} 397}$ bands. Intensity values of each phosphorylated protein were adjusted to the corresponding PP2A and WAVE1 intensity values and then normalized to the control. ${ }^{*} \mathrm{p}<0.05$ vs. CON. I) Dendritic spine density analysis. Cells were counterstained with Texas Red phalloidin to visualize actin filaments. The scale bar corresponds to $5 \mu \mathrm{m}$. The graph shows the mean value $\pm \mathrm{SD}$ of the number of spines in $10 \mu \mathrm{m}$ of dendrite length (20-30 neurons from three independent experiments were analyzed per condition). ${ }^{*} \mathrm{p}<0.05$ vs. CON.

Figure 4. Expression of PP2A mutant constructs affects DS formation. The mutant constructs of PP2A (PP2A $\mathrm{A}^{\mathrm{Y} 307 \mathrm{E}}$ and $\left.\mathrm{PP} 2 \mathrm{~A}^{\mathrm{Y} 307 \mathrm{~F}}\right)$ were transfected into cortical neuronal cells and incubated in the presence of E2 and P4 (10 nM) for 20 min. A) Actin, phosphoWAVE1 ${ }^{\mathrm{S} 397}$ and PP2A were assayed in cell extracts. B-C) The densitometry values were adjusted to actin intensity, then normalized to the control sample. ${ }^{*} \mathrm{p}<0.05$ vs. corresponding control. D-E) Analysis of dendritic spine density. Note that in the PP2A DN group, E2 and P4 PP2A DN increased the number of DS, although the difference was not statistically significant. Cells were counterstained with Texas Red phalloidin to visualize actin filaments. The scale bar corresponds to $5 \mu \mathrm{m}$. The graph shows the mean value $\pm \mathrm{SD}$ of the number of spines in $10 \mu \mathrm{m}$ of dendrite length (20-30 neurons). CON, Control. F) Cells were exposed to E2 and P4 for $20 \mathrm{~min}$ in the presence or absence of PP2 $(10 \mu \mathrm{M})$, and Src, PP2A and cortactin phosphorylation were analyzed through Western blot assay. G-I) Phospho-Src ${ }^{\text {Y418 }}$ p-PP2 $\mathrm{A}^{\mathrm{Y} 307}$ and cortactin $^{\mathrm{Y} 466}$ densitometry values were adjusted to actin intensity and normalized to the control. All experiments were performed in triplicate with consistent results; representative images are shown. ${ }^{*} \mathrm{p}<0.05$ vs. CON. 
Figure 5. E2 and P4 mediated cortactin phosphorylation and redistribution via Rac1/PAK1 in rat cortical neurons. Neuron cells were treated with E2 (10 nM) and P4 (10 $\mathrm{nM})$ for $20 \mathrm{~min}$ in the presence or absence of IPA-3 for $45 \min (0,5 \mu \mathrm{M})$ and transfected with Rac1DN and cortactin ${ }^{3 \mathrm{YF}}$ mutant constructs. A) Expression of phosphorylated Rac1 ${ }^{\mathrm{S} 71}$ and PAK1 $1^{\mathrm{T} 423}$ was analyzed with Western blot. B-C) Densitometric analysis of p-Rac1 ${ }^{\mathrm{S} 71}$ and $\mathrm{p}-\mathrm{PAK} 1^{\mathrm{T} 423}$ bands. Intensity values of each protein were adjusted to the corresponding intensity values of actin and then normalized to the control sample. ${ }^{*} \mathrm{p}<0.05$ vs. control, CON. D) Quantification of the cell membrane dendrite-localized phospho-cortactin ${ }^{\mathrm{Y} 466}$ in the different conditions. E) Mean \pm SD of the pixel intensity of phospho-cortactin ${ }^{\text {Y466 }}$ vs. CON. $* \mathrm{p}<0.05$ vs. CON. Dendrite-localized phospho-cortactin ${ }^{\mathrm{Y} 466}$ was counted in 30 different cells. F) Dendritic spine density was measured with immunofluorescence after staining actin fibers with phalloidin/Texas Red. The scale bar corresponds to $5 \mu \mathrm{m}$. G) Quantitative analysis of spine density expressed as the number of spines per $10 \mu \mathrm{m}$ dendrite length. ${ }^{*} \mathrm{p}<0.05$ vs. CON. The experiments were repeated three times with consistent results.

Figure 6. E2 and P4 may modulate the molecular interaction between cortactin and Arp2/3 complex in cortical neuronal cells. Neurons were treated with A-B) E2 (10 nM) or C-D) P4 (10 nM), both for $20 \mathrm{~min}$, and the cell protein extracts immunoprecipitated (IPs) with an antibody vs. Arp3. IPs were assayed for co-immunoprecipitation of cortactin and Arp3. Membranes were re-blotted for the immunoprecipitated protein to show equal loading. E-H) Cells were treated for 20 min with (E-F) E2 (10 nM) or (G-H) P4 (10 nM) after $\operatorname{cortactin}^{3 \mathrm{YF}}$ transfection and tested by Western blot. Phosphorylated Arp2 ${ }^{\text {Thr237 }}$ and actin 
were analyzed in the cell protein extracts. ${ }^{*} p<0.05$ vs. CON. The experiments were performed in triplicate; representative images are shown. CON, Control.

Figure 7. Cortactin and WAVE1 control DS formation through the regulation of Arp2/3 complex. A) Cortical neurons were treated with E2 (10 nM) and P4 (10 nM) for 20 min in the presence or absence of $\operatorname{cortactin}^{3 \mathrm{YF}}$ plasmid. Total cell amount of wild-type cortactin, $\mathrm{p}$-cortactin ${ }^{\mathrm{Y} 466}, \mathrm{p}$-WAVE1 ${ }^{\mathrm{S} 397}, \mathrm{p}$-Arp2 ${ }^{\mathrm{T} 237}$ and actin are shown with Western blot. B-E) Phospho-cortactin ${ }^{\mathrm{Y} 466}, \mathrm{p}-\mathrm{WAVE} 1^{\mathrm{S} 397}$ and $\mathrm{p}$-Arp2 $2^{\mathrm{T} 237}$ densitometry values were adjusted to actin intensity, respectively, then normalized to the control sample. F-J) Cortical cells were transfected with siRNAs vs. WAVE1, and protein analysis for WAVE1, pWAVE1 ${ }^{\mathrm{S} 397}, \mathrm{p}$-cortactin ${ }^{\mathrm{Y} 466}, \mathrm{p}$-Arp2 $2^{\mathrm{T} 237}$ and actin was performed on cell lysates after treatment for 20 min with E2 and P4 (both 10 nM). Phospho-WAVE1 ${ }^{\text {S397, }}$, p-cortactin ${ }^{\text {Y466 }}$ and $\mathrm{p}$-Arp $2^{\mathrm{T} 237}$ densitometry values were adjusted to actin intensity, then normalized to the control sample. ${ }^{*} \mathrm{p}<0.05$ vs. corresponding control.

Figure 8. Arp2/3 complex is involved in the final step to promote DS formation induced by E2 and P4. Cortical cells were exposed for $20 \mathrm{~min}$ to $\mathrm{E} 2(10 \mathrm{nM})$ and P4 (10 nM) in the presence or absence of the Arp 2/3 complex inhibitor CK-666 $(4 \mu \mathrm{M})$. A) Actin and phospho-Arp2 $2^{\mathrm{T} 237}$ were analyzed through Western blot assay. B) Phospho-Arp2 $2^{\mathrm{T} 237}$ densitometry value was adjusted to actin intensity, then normalized to the control sample. CON, Control. All experiments were performed three times and representative blots are presented. ${ }^{*} \mathrm{p}<0.05$ vs. CON. C) Dendritic spine density analysis. Cells were counterstained with Texas Red-phalloidin to visualize actin filaments. The scale bar corresponds to $5 \mu \mathrm{m}$. D) Mean $\pm \mathrm{SD}$ of the number of spines in $10 \mu \mathrm{m}$ of dendrite length (20-30 neurons) from 
three independent experiments per condition. ${ }^{*} \mathrm{p}<0.05$ vs. CON. E) Cortical neurons were exposed for 20 min to E2 and P4 in the presence or absence of the ER/PR antagonists ICI 182,780 and ORG 31710. Phosphorylation of PP2A, WAVE1 and Arp2 were assayed with Western analysis. F) Densitometric analysis of p-PP2A1 ${ }^{\mathrm{Y} 307}$, p-WAVE1 $^{\mathrm{S} 397}$ and p-Arp2 ${ }^{\mathrm{T} 237}$ bands. Intensity values of each protein were adjusted to the corresponding intensity values of actin and then normalized to the control sample. ${ }^{*} \mathrm{p}<0.05$ vs. CON.

Figure 9. Representative intracellular signaling of E2 and P4 to cortactin/WAVE1Arp2/3 complex implicated in the control of DS formation. Novel mechanism triggered by E2 and P4 regulates actin nucleation via cortactin and WAVE1 and their target protein Arp2/3 complex, allowing a rapid formation of dendritic spines in rat cortical neuronal cells. 


\section{ACKNOWLEDGEMENTS}

We thank Dr. Mariella Superina for correcting this manuscript. The authors are grateful to Juan Rosales for his excellent technical assistance and to Dr. S. R. Valdez for the Synaptophysin antibody.

DISCLOSURE STATEMENT: The authors have nothing to disclose.

\section{FUNDING}

This work has been supported by National Scientific and Technical Research Council (CONICET) grant PIP 2013-2015 and by the National Agency for Scientific and Technological Promotion (The Ministry of Science, Technology and Productive Innovation) PICT-2013-3230 to A.M.S.

\section{AUTHORS CONTRIBUTION STATEMENT}

IDU carried out different experiments, cell culture and treatments.

MIF performed immunofluorescence assays and participated to the writing of the MS.

AMS planned and funded the project, supervised the experiments, wrote the paper. 


\section{REFERENCES}

1. Kasai, H., et al., Structural dynamics of dendritic spines in memory and cognition. Trends Neurosci, 2010. 33(3): p. 121-9.

2. Penzes, P., et al., Dendritic spine pathology in neuropsychiatric disorders. Nat Neurosci, 2011. 14(3): p. 285-93.

3. McEwen, B.S. and T.A. Milner, Understanding the broad influence of sex hormones and sex differences in the brain. J Neurosci Res, 2017. 95(1-2): p. 24-39.

4. Hansberg-Pastor, V., et al., Sex Hormones Regulate Cytoskeletal Proteins Involved in Brain Plasticity. Front Psychiatry, 2015. 6: p. 165.

5. Arevalo, M.A., et al., Signaling mechanisms mediating the regulation of synaptic plasticity and memory by estradiol. Horm Behav, 2015. 74: p. 19-27.

6. Phan, A., et al., Low doses of 17beta-estradiol rapidly improve learning and increase hippocampal dendritic spines. Neuropsychopharmacology, 2012. 37(10): p. 2299-309.

7. Sakamoto, H., K. Ukena, and K. Tsutsui, Dendritic spine formation in response to progesterone synthesized de novo in the developing Purkinje cell in rats. Neurosci Lett, 2002. 322(2): p. 111-5.

8. Frankfurt, M. and V. Luine, The evolving role of dendritic spines and memory: Interaction(s) with estradiol. Horm Behav, 2015. 74: p. 28-36.

9. Melcangi, R.C., et al., Levels and actions of progesterone and its metabolites in the nervous system during physiological and pathological conditions. Prog Neurobiol, 2014. 113: p. 5669.

10. Sherwin, B.B. and J.F. Henry, Brain aging modulates the neuroprotective effects of estrogen on selective aspects of cognition in women: a critical review. Front Neuroendocrinol, 2008. 29(1): p. 88-113.

11. Hlushchenko, I., M. Koskinen, and P. Hotulainen, Dendritic spine actin dynamics in neuronal maturation and synaptic plasticity. Cytoskeleton (Hoboken), 2016. 73(9): p. 435-41.

12. Helgeson, L.A., et al., Interactions with actin monomers, actin filaments, and Arp2/3 complex define the roles of WASP family proteins and cortactin in coordinately regulating branched actin networks. J Biol Chem, 2014. 289(42): p. 28856-69.

13. Zhang, J., et al., Phosphorylation and SUMOylation of CRMP2 regulate the formation and maturation of dendritic spines. Brain Res Bull, 2018. 139: p. 21-30.

14. Lee, Y., et al., Phosphorylation of CYFIP2, a component of the WAVE-regulatory complex, regulates dendritic spine density and neurite outgrowth in cultured hippocampal neurons potentially by affecting the complex assembly. Neuroreport, 2017. 28(12): p. 749-754.

15. Ceglia, I., et al., Signaling pathways controlling the phosphorylation state of WAVE1, a regulator of actin polymerization. J Neurochem, 2010. 114(1): p. 182-90.

16. Young, M.R., K. Kolesiak, and J. Meisinger, Protein phosphatase-2A regulates endothelial cell motility and both the phosphorylation and the stability of focal adhesion complexes. Int J Cancer, 2002. 100(3): p. 276-82.

17. Winder, D.G. and J.D. Sweatt, Roles of serine/threonine phosphatases in hippocampal synaptic plasticity. Nat Rev Neurosci, 2001. 2(7): p. 461-74.

18. Hering, H. and M. Sheng, Activity-dependent redistribution and essential role of cortactin in dendritic spine morphogenesis. J Neurosci, 2003. 23(37): p. 11759-69.

19. Daly, R.J., Cortactin signalling and dynamic actin networks. Biochem J, 2004. 382(Pt 1): p. 13-25.

20. Weaver, A.M., et al., Interaction of cortactin and N-WASp with Arp2/3 complex. Curr Biol, 2002. 12(15): p. 1270-8.

21. Sanchez, A.M., et al., Effects of progesterone and medroxyprogesterone on actin remodeling and neuronal spine formation. Mol Endocrinol, 2013. 27(4): p. 693-702. 
22. Sanchez, A.M., et al., Rapid signaling of estrogen to WAVE1 and moesin controls neuronal spine formation via the actin cytoskeleton. Mol Endocrinol, 2009. 23(8): p. 1193-202.

23. Kaech, S. and G. Banker, Culturing hippocampal neurons. Nat Protoc, 2006. 1(5): p. 2406-15.

24. Schubert, V. and C.G. Dotti, Transmitting on actin: synaptic control of dendritic architecture. J Cell Sci, 2007. 120(Pt 2): p. 205-12.

25. Rodriguez, A., et al., Automated three-dimensional detection and shape classification of dendritic spines from fluorescence microscopy images. PLoS One, 2008. 3(4): p. e1997.

26. Tehrani, S., et al., Src phosphorylation of cortactin enhances actin assembly. Proc Natl Acad Sci U S A, 2007. 104(29): p. 11933-8.

27. Maiti, P., et al., Molecular regulation of dendritic spine dynamics and their potential impact on synaptic plasticity and neurological diseases. Neurosci Biobehav Rev, 2015. 59: p. 20837.

28. Moeller, M.L., et al., EphB receptors regulate dendritic spine morphogenesis through the recruitment/phosphorylation of focal adhesion kinase and RhoA activation. J Biol Chem, 2006. 281(3): p. 1587-98.

29. Chen, J., B.L. Martin, and D.L. Brautigan, Regulation of protein serine-threonine phosphatase type-2A by tyrosine phosphorylation. Science, 1992. 257(5074): p. 1261-4.

30. Liu, R. and J.Z. Wang, Protein phosphatase 2A in Alzheimer's disease. Pathophysiology, 2009. 16(4): p. 273-7.

31. Sontag, J.M. and E. Sontag, Protein phosphatase 2A dysfunction in Alzheimer's disease. Front Mol Neurosci, 2014. 7: p. 16.

32. Vidal, C., et al., Cdc42/Rac1-dependent activation of the p21-activated kinase (PAK) regulates human platelet lamellipodia spreading: implication of the cortical-actin binding protein cortactin. Blood, 2002. 100(13): p. 4462-9.

33. Yin, M., W. Ma, and L. An, Cortactin in cancer cell migration and invasion. Oncotarget, 2017. 8(50): p. 88232-88243.

34. Vicente-Manzanares, M., J. Hodges, and A.R. Horwitz, Dendritic Spines: Similarities with Protrusions and Adhesions in Migrating Cells. Open Neurosci J, 2009. 3: p. 87-96.

35. Moshfegh, Y., et al., A Trio-Rac1-Pak1 signalling axis drives invadopodia disassembly. Nat Cell Biol, 2014. 16(6): p. 574-86.

36. Jeannot, P. and A. Besson, Cortactin function in invadopodia. Small GTPases, 2017: p. 1-15.

37. Tegtmeyer, N., et al., Serine phosphorylation of cortactin controls focal adhesion kinase activity and cell scattering induced by Helicobacter pylori. Cell Host Microbe, 2011. 9(6): p. 520-31.

38. Racz, B. and R.J. Weinberg, The subcellular organization of cortactin in hippocampus. J Neurosci, 2004. 24(46): p. 10310-7.

39. Uruno, T., et al., Activation of Arp2/3 complex-mediated actin polymerization by cortactin. Nat Cell Biol, 2001. 3(3): p. 259-66.

40. Narayanan, A., et al., Phosphorylation of the Arp2 subunit relieves auto-inhibitory interactions for Arp2/3 complex activation. PLoS Comput Biol, 2011. 7(11): p. e1002226.

41. LeClaire, L.L., 3rd, et al., Phosphorylation of the Arp2/3 complex is necessary to nucleate actin filaments. J Cell Biol, 2008. 182(4): p. 647-54.

42. Wegner, A.M., et al., $N$-wasp and the arp2/3 complex are critical regulators of actin in the development of dendritic spines and synapses. J Biol Chem, 2008. 283(23): p. 15912-20.

43. Spillane, M., et al., The actin nucleating Arp2/3 complex contributes to the formation of axonal filopodia and branches through the regulation of actin patch precursors to filopodia. Dev Neurobiol, 2011. 71(9): p. 747-58.

44. Rochefort, N.L. and A. Konnerth, Dendritic spines: from structure to in vivo function. EMBO Rep, 2012. 13(8): p. 699-708.

45. Duffau, H., Brain plasticity: from pathophysiological mechanisms to therapeutic applications. J Clin Neurosci, 2006. 13(9): p. 885-97. 
46. Gipson, C.D. and M.F. Olive, Structural and functional plasticity of dendritic spines - root or result of behavior? Genes Brain Behav, 2017. 16(1): p. 101-117.

47. Bourne, J. and K.M. Harris, Do thin spines learn to be mushroom spines that remember? Curr Opin Neurobiol, 2007. 17(3): p. 381-6.

48. Smith, C.C., L.C. Vedder, and L.L. McMahon, Estradiol and the relationship between dendritic spines, NR2B containing NMDA receptors, and the magnitude of long-term potentiation at hippocampal CA3-CA1 synapses. Psychoneuroendocrinology, 2009. 34 Suppl 1: p. S130-42.

49. Lambrecht, C., et al., Structure, regulation, and pharmacological modulation of PP2A phosphatases. Methods Mol Biol, 2013. 1053: p. 283-305.

50. Liu, R., et al., Phosphorylated PP2A (tyrosine 307) is associated with Alzheimer neurofibrillary pathology. J Cell Mol Med, 2008. 12(1): p. 241-57.

51. Li, D., et al., Striatin-1 is a B subunit of protein phosphatase PP2A that regulates dendritic arborization and spine development in striatal neurons. J Biol Chem, 2018. 293(28): p. 11179-11194.

52. Lin, L., et al., Determination of dendritic spine morphology by the striatin scaffold protein STRN4 through interaction with the phosphatase PP2A. J Biol Chem, 2017. 292(23): p. 94519464.

53. Liu, X., et al., PRG-1 Regulates Synaptic Plasticity via Intracellular PP2A/beta1-Integrin Signaling. Dev Cell, 2016. 38(3): p. 275-90.

54. Soderling, S.H., et al., Loss of WAVE-1 causes sensorimotor retardation and reduced learning and memory in mice. Proc Natl Acad Sci U S A, 2003. 100(4): p. 1723-8.

55. Bhambhvani, H.P., et al., Decreased expression of cortactin in the schizophrenia brain. Neuroreport, 2016. 27(3): p. 145-50.

56. Kim, I.H., et al., Disruption of Arp2/3 results in asymmetric structural plasticity of dendritic spines and progressive synaptic and behavioral abnormalities. J Neurosci, 2013. 33(14): p. 6081-92. 
Fig. $1 \mathrm{~A}$
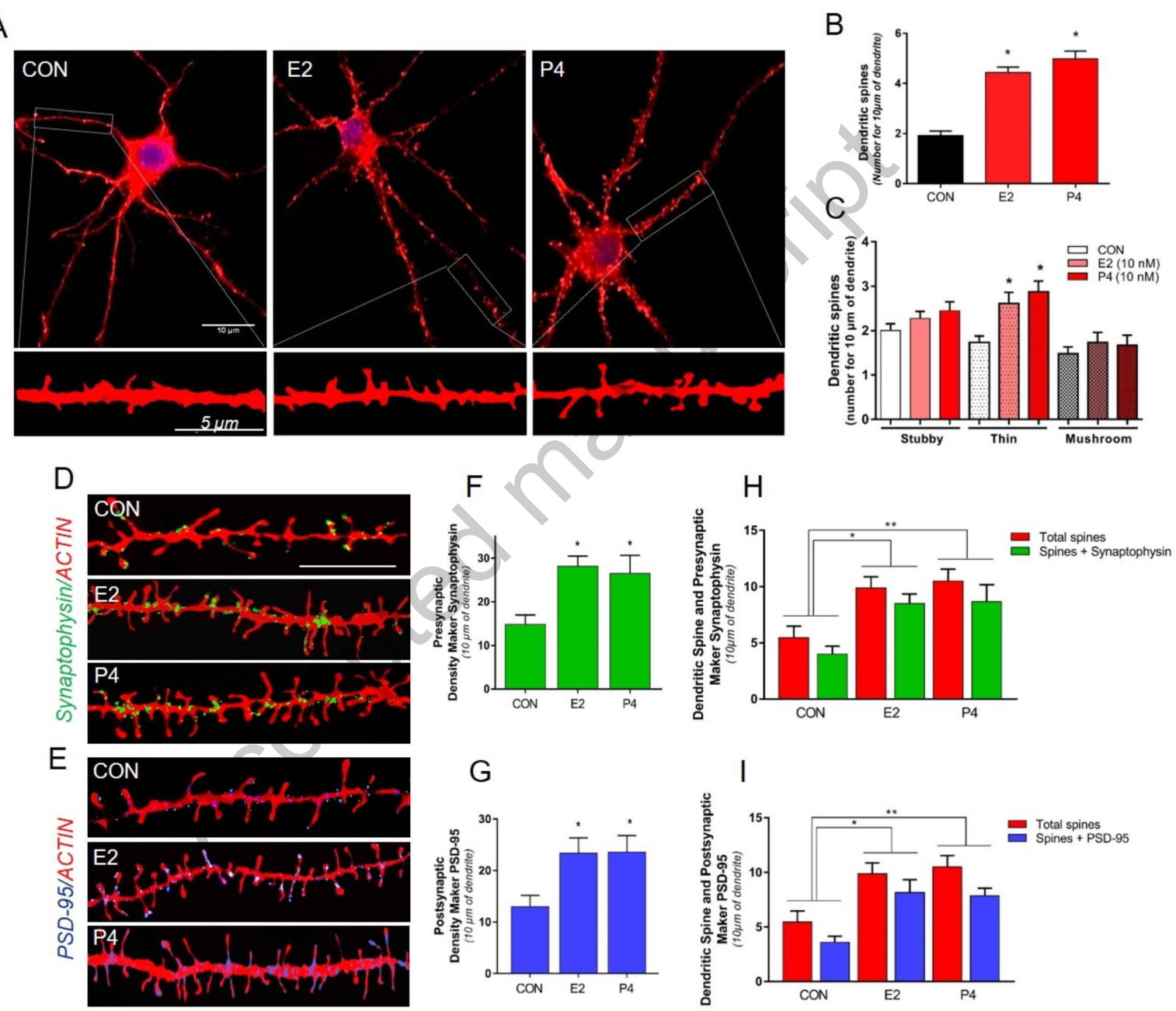
Fig. 2

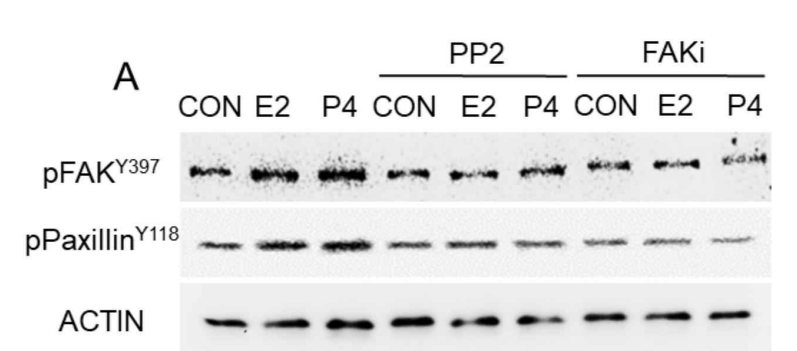

B
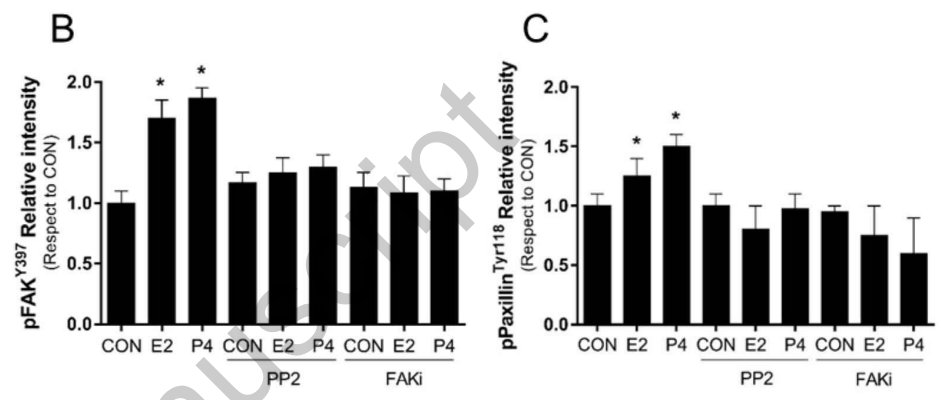

E

F
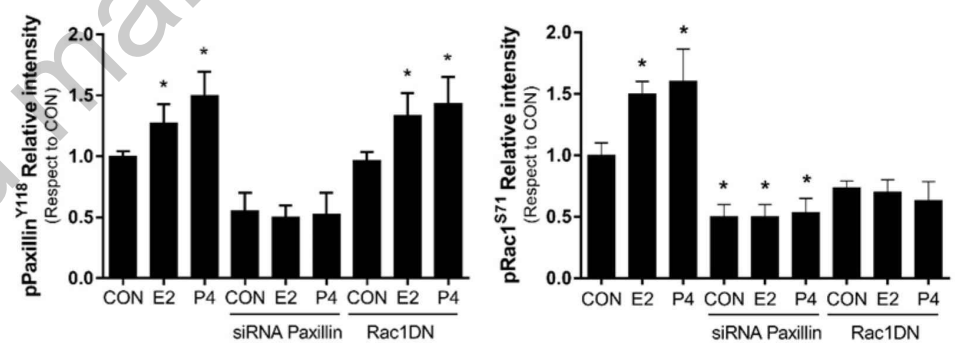

G

SiRNA Paxillin CON E2 P4 CON E2 P4
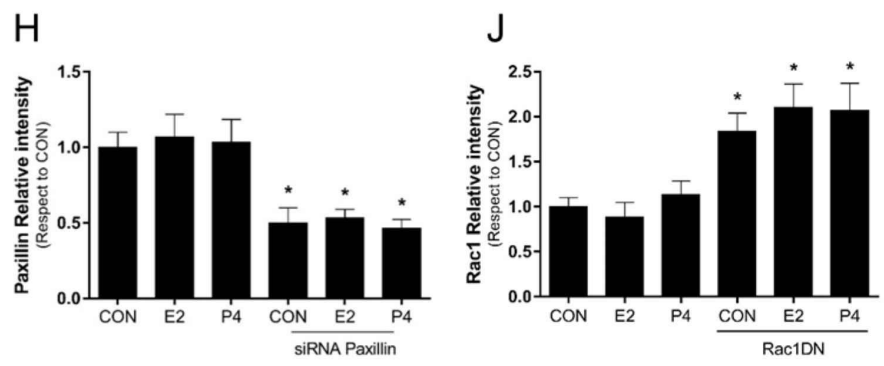
Rac1 


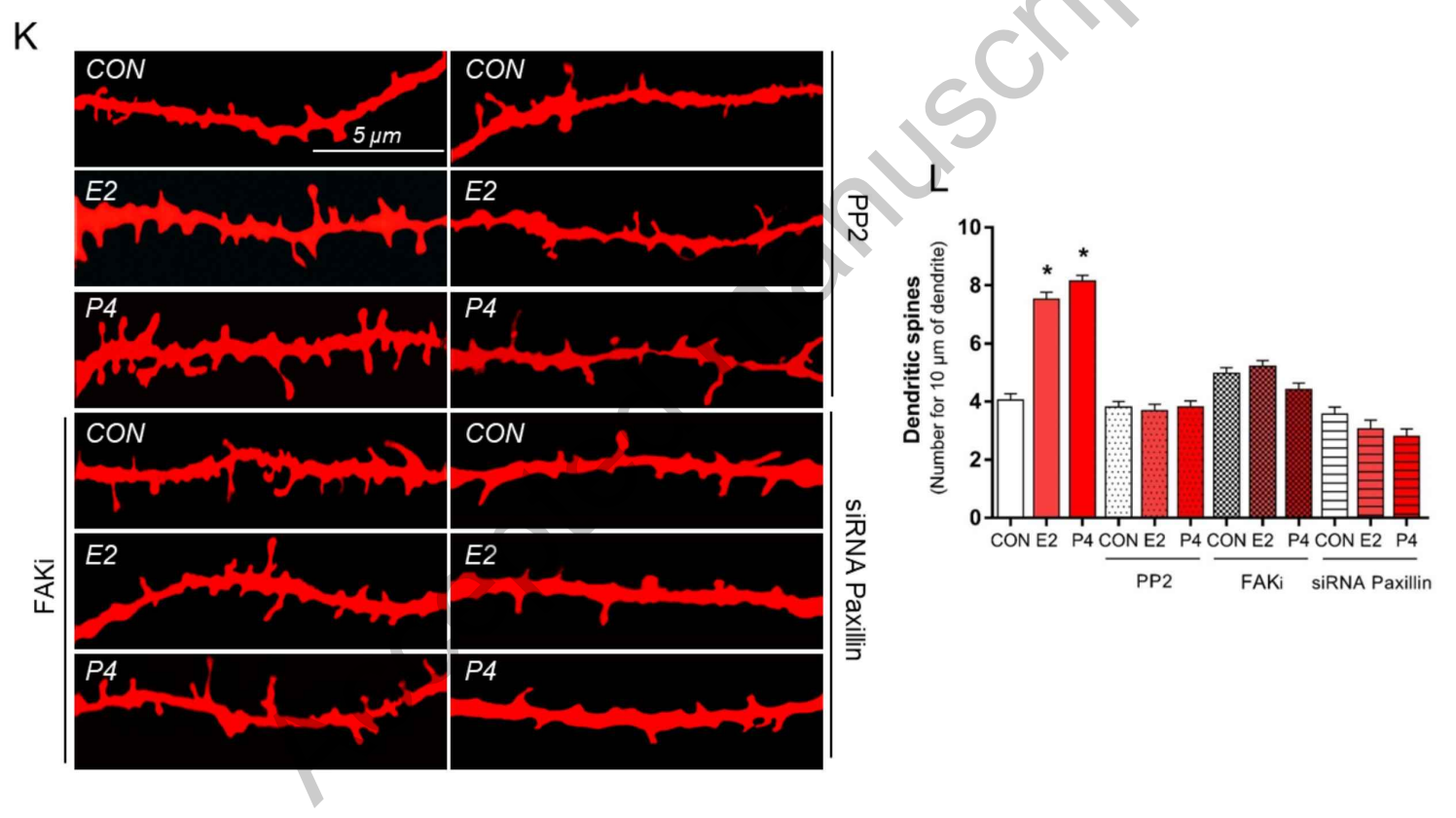


Fig. 3

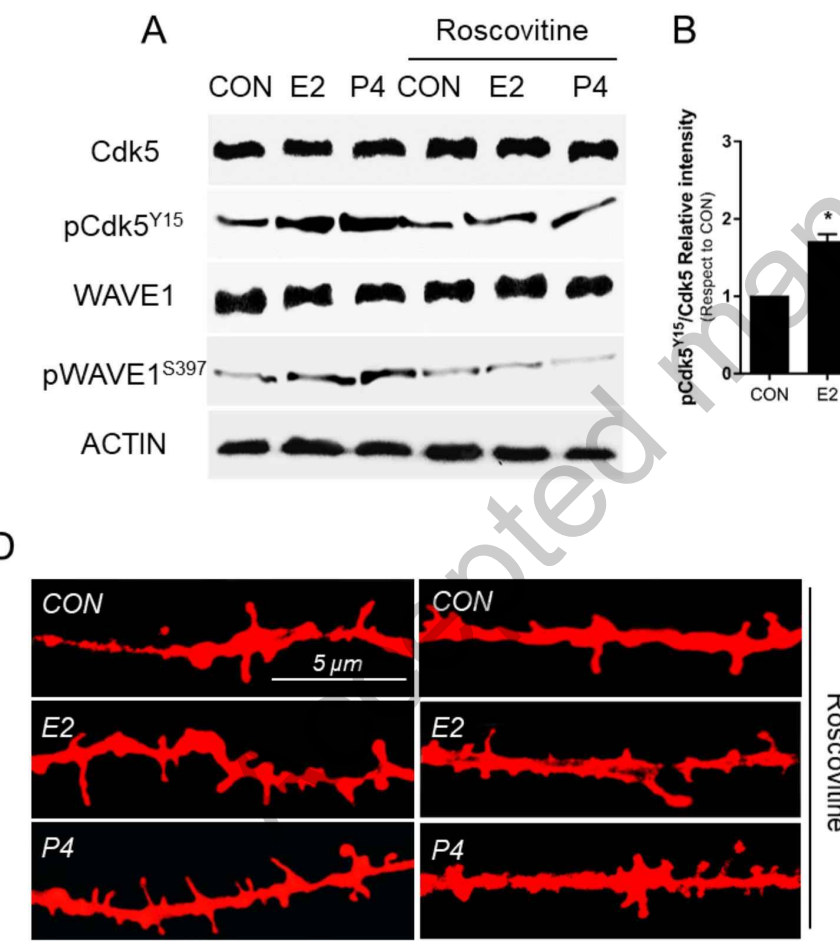

C 


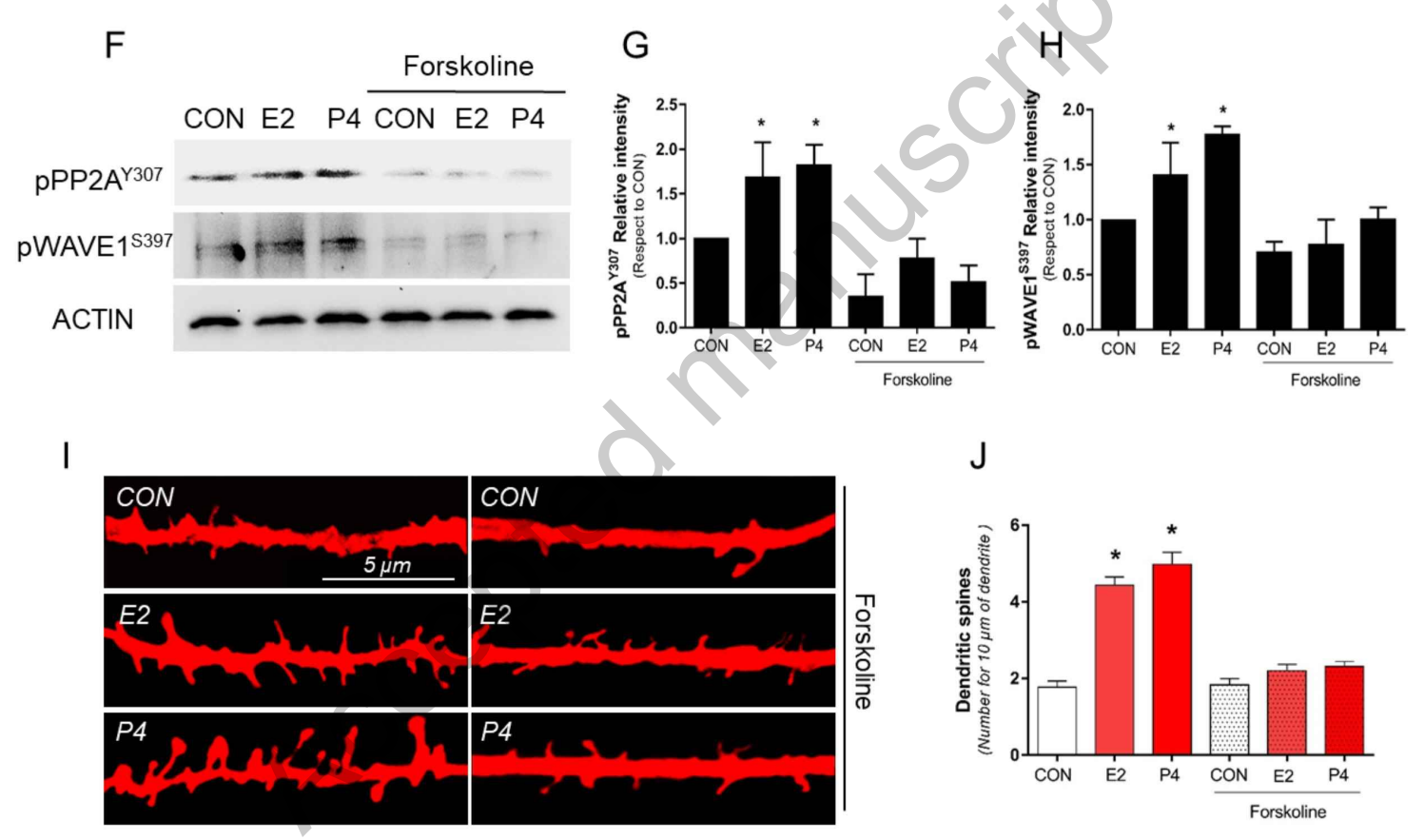


Fig. 4

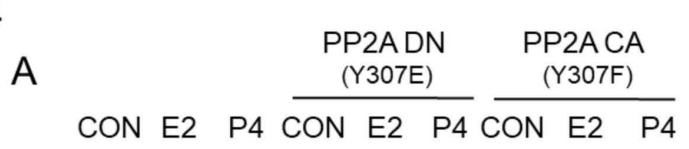

PP2A

PWAVE $1^{\text {S397 }}$

ACTIN

D

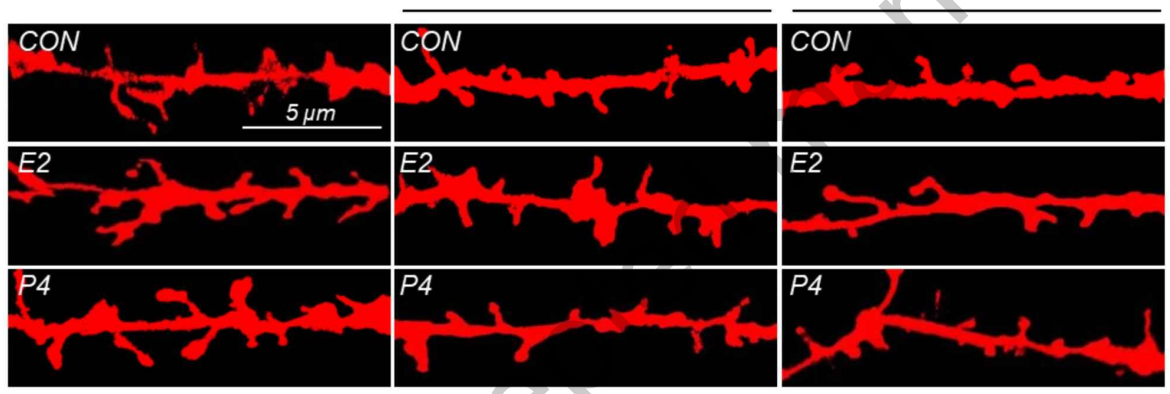

C

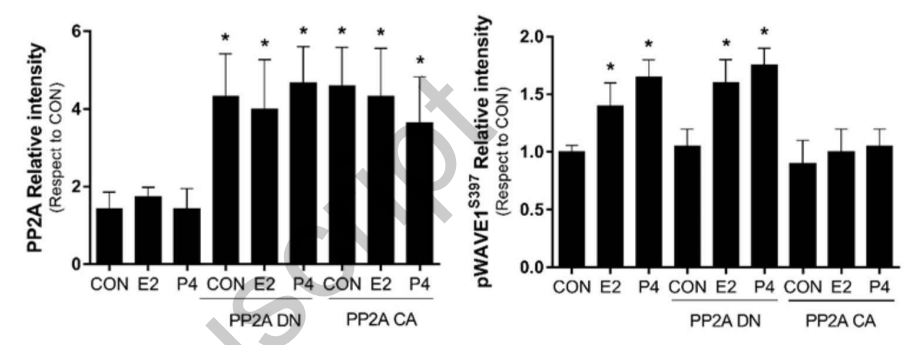

E

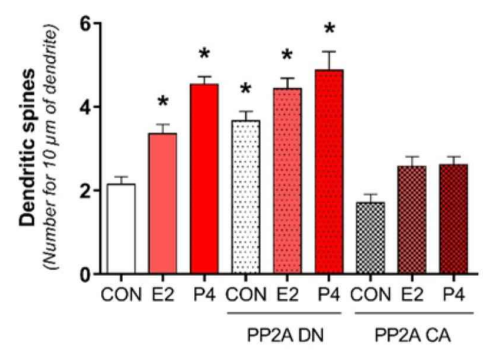

F CON E2 P4 $\frac{\text { PP2 }}{\text { CON E2 P4 }}$

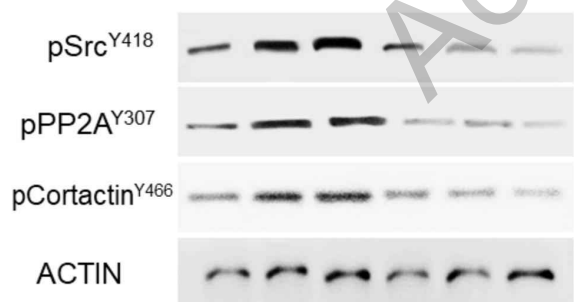

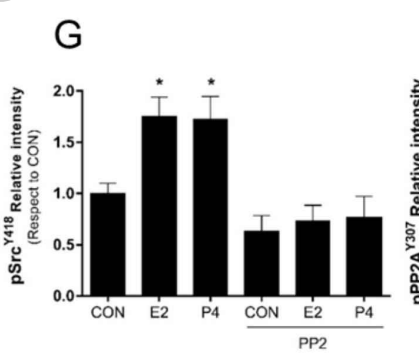

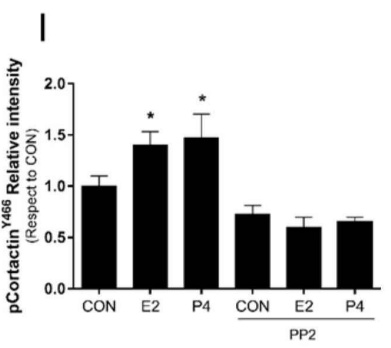


Fig. 5

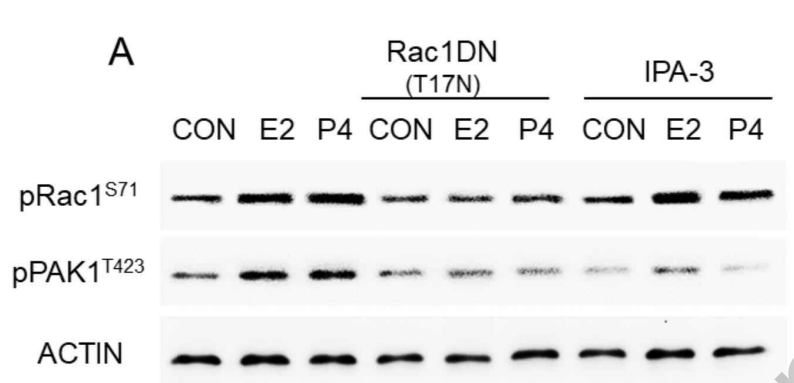

B C
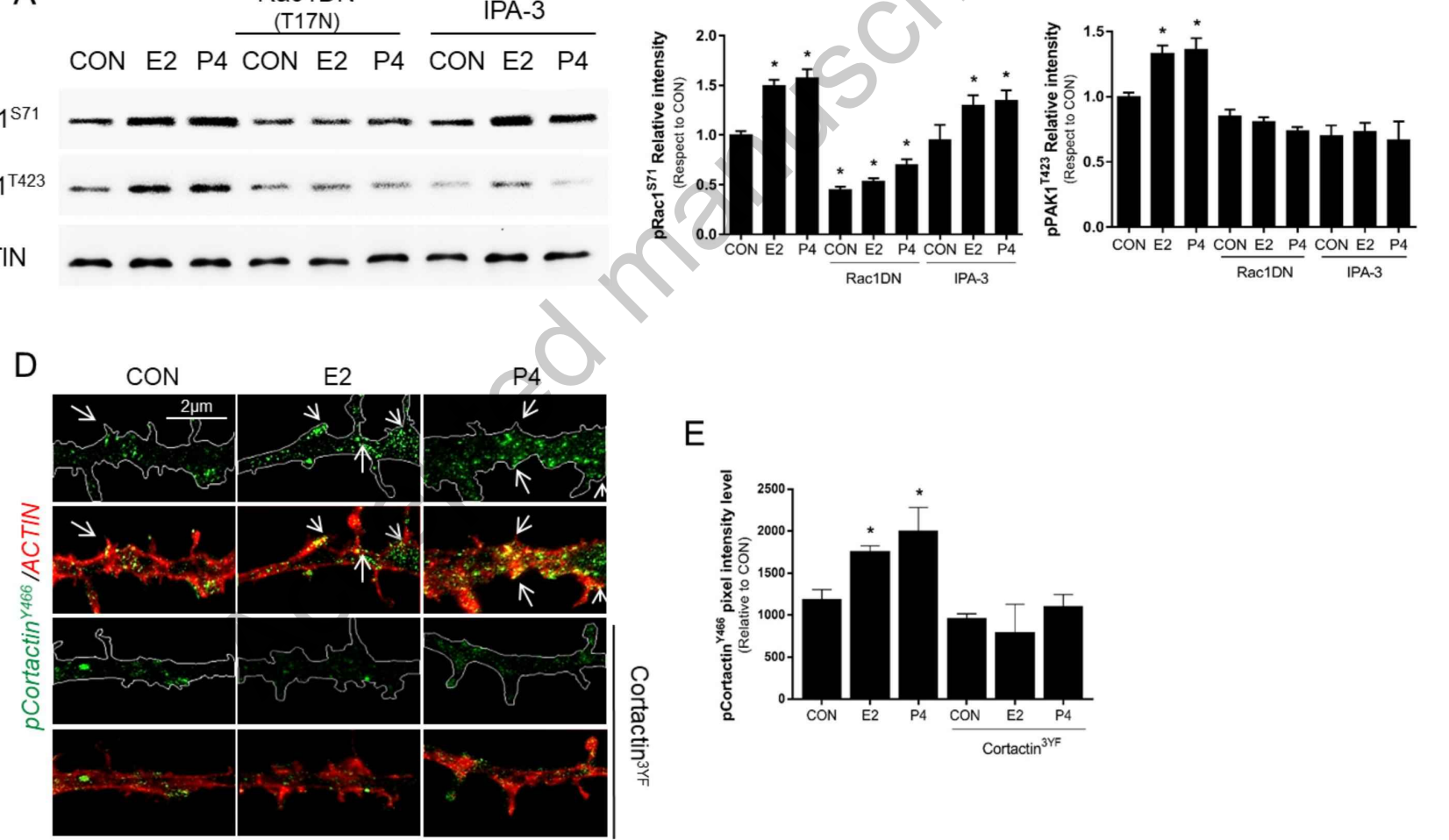


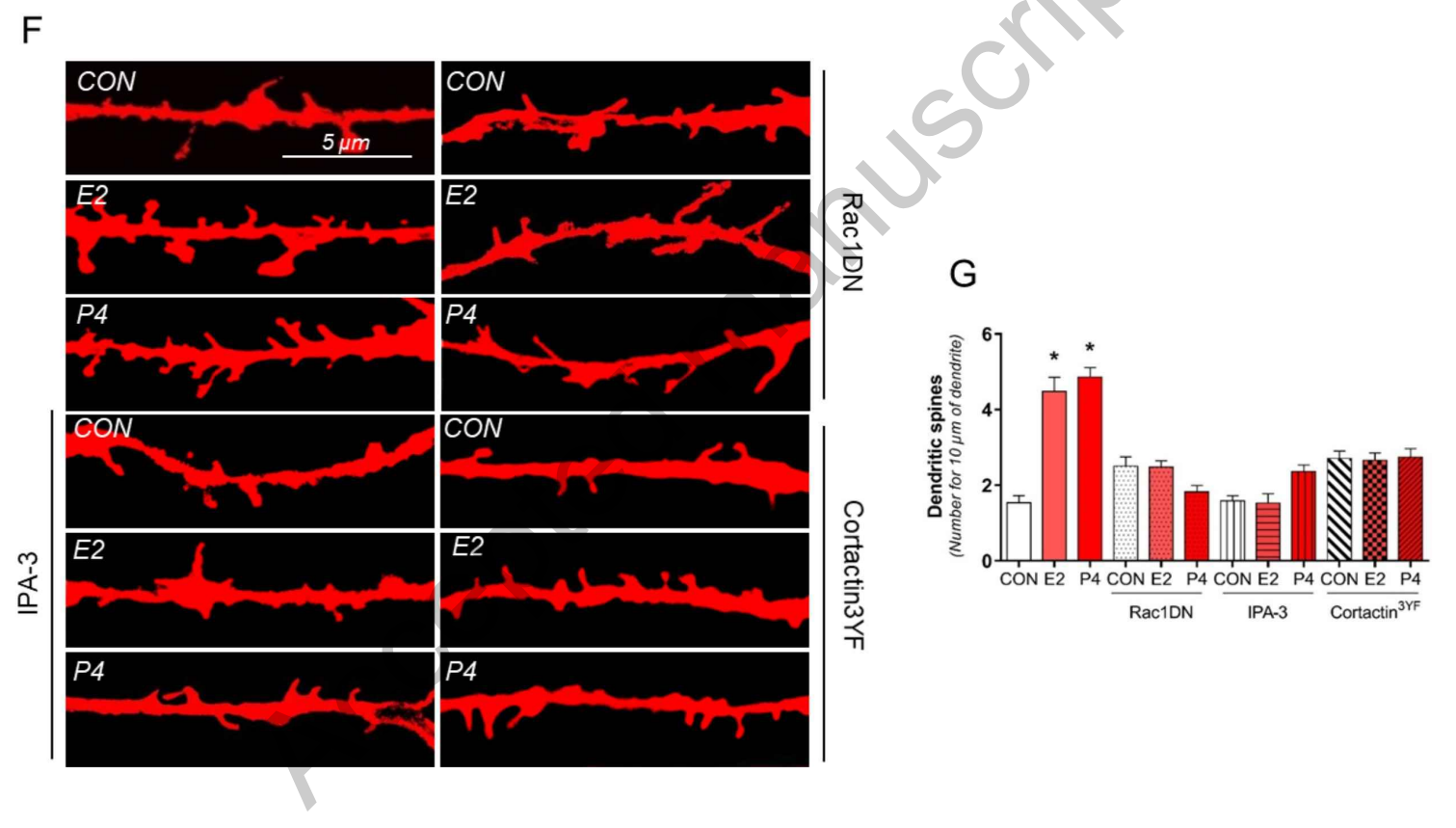


Fig. 6

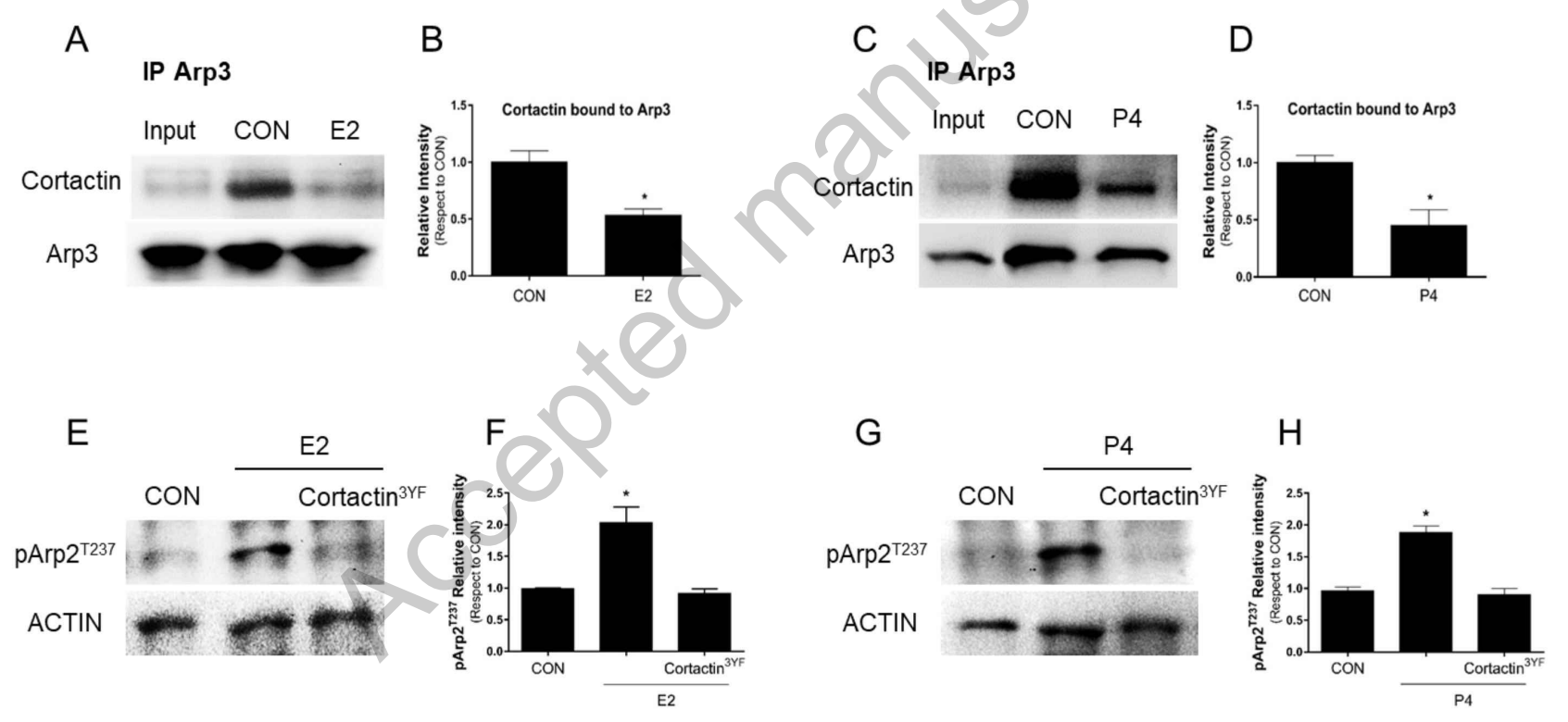


Fig. 7

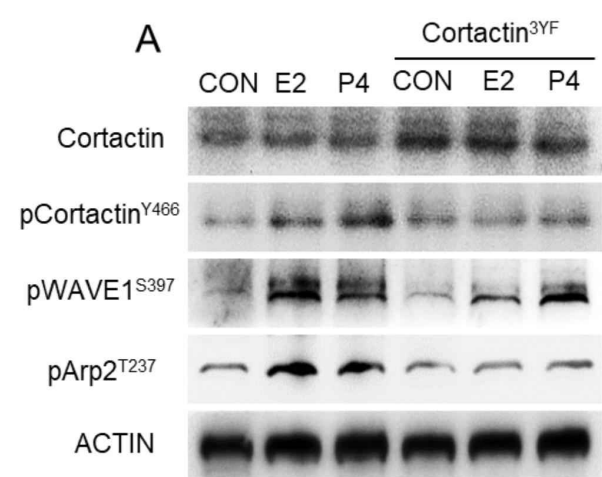

B

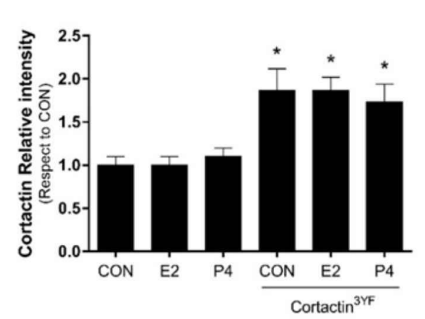

D

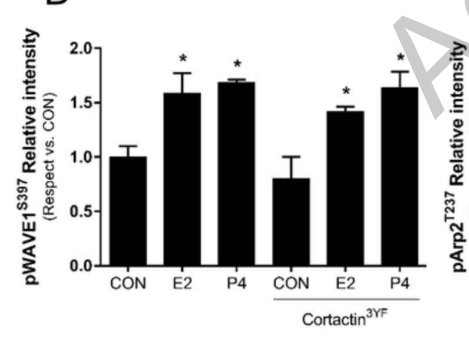

C

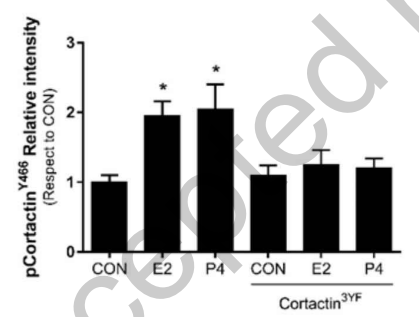

E

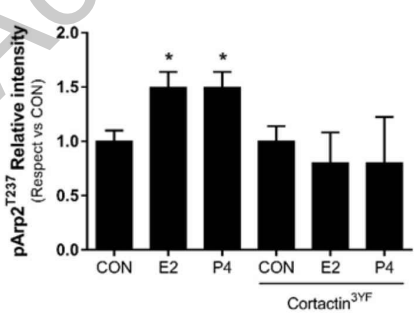

F CON E2 P4 CON E2 P4

WAVE1

PWAVE1539

pCortactin ${ }^{\curlyvee 466}$

pArp2 $^{\text {T237 }}$

ACTIN
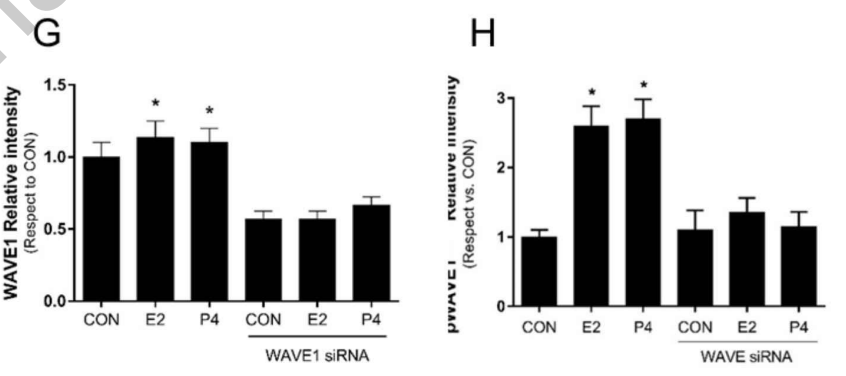

J
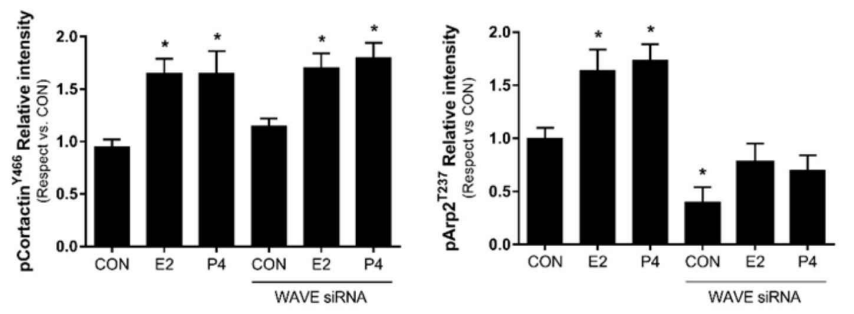
Fig. 8

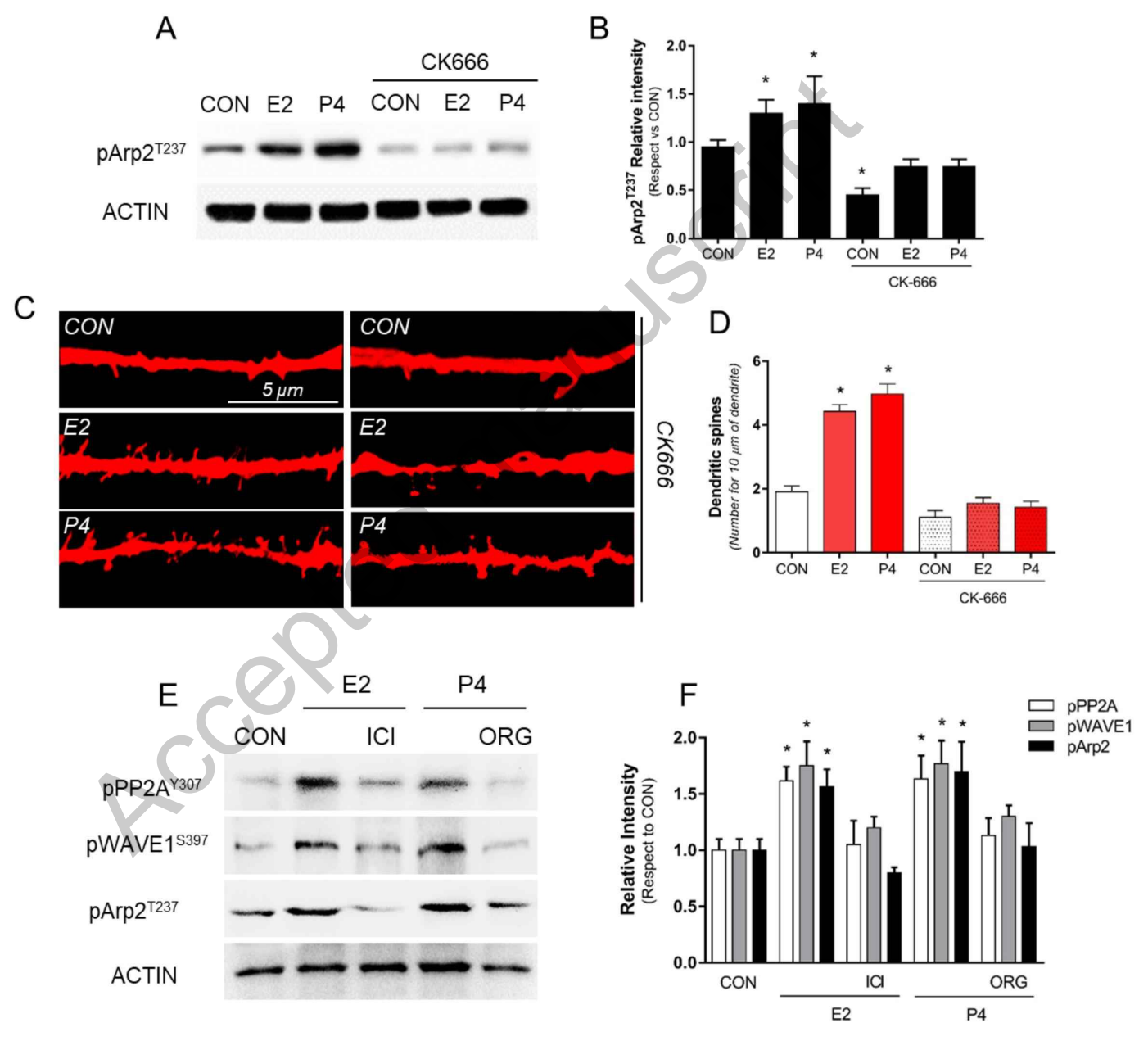


Fig. 9

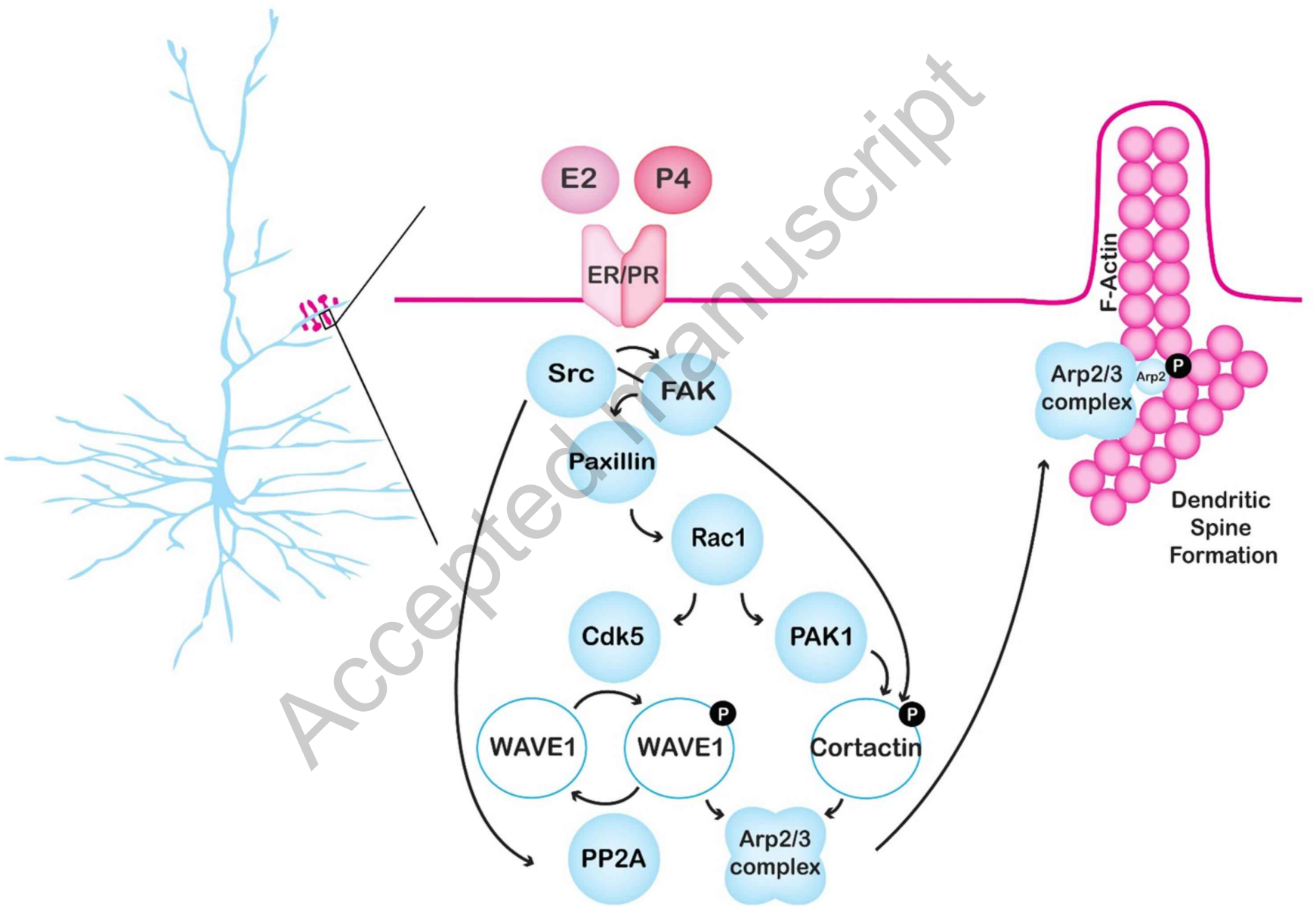

